Algebra univers. 57 (2007) 333-370

0002-5240/07/030333 - 38, published online November 5, 2007

DOI 10.1007/s00012-007-2055-8

(C) Birkhäuser Verlag, Basel, 2007

Algebra Universalis

\title{
Representable semilattice-ordered monoids
}

\author{
Robin HiRsch And Szabolcs Mikulás
}

Abstract. We show that no finite set of first-order axioms can define the class of representable semilattice-ordered monoids.

\section{Introduction}

Relation Algebra is an algebraic abstraction of the study of binary relations. Tarski showed that Relation Algebra can act as a vehicle for set theory and hence all of mathematics, cf. [20]. He also showed that the classes of relation algebras (RA) and representable relation algebras (RRA) are equational varieties [19]. Monk proved that RRA is indeed a canonical variety (reported in [15, Theorem 2.12]). On the other hand, relation algebras are badly behaved in a number of ways: RRA cannot be defined by finitely many axioms [17]; the equational theories of RA and RRA are not decidable $[18,20,3]$; RRA cannot be defined by any set of canonical equations [13] nor by any set of equations using only finitely many variables [14]; the problem of determining whether a finite relation algebra is representable is an undecidable problem [10]. An important line of research is to restrict the signature of relation algebras and study the behaviour of the corresponding representation class. We are interested to see if the representation class is well behaved in some way: does the class form an equational variety or, if not, a quasi-variety; is its equational theory decidable; is it finitely axiomatisable; etc.?

The signature of relation algebra is that of boolean algebra (BA) $\{0,1,+, \cdot,-\}$ together with extra operators $\left\{1^{\prime}, \smile, ;\right\}$. If $S \subseteq\left\{0,1,+, \cdot,-, 1^{\prime}, \smile, ;\right\}$ then the representation class $R(S)$ consists of those algebras $\mathcal{A}$ in this signature for which there is an injection $h: \mathcal{A} \rightarrow \wp(U)$ (the power set of $U$ ) where $U \subseteq D \times D$ (for some base set $D$ ) which respects those boolean operations in $S$, and

$$
\begin{aligned}
h\left(1^{\prime}\right) & =\{(d, d): d \in D\} \\
h\left(a^{\smile}\right) & =\left\{\left(d, d^{\prime}\right):\left(d^{\prime}, d\right) \in h(a)\right\} \\
h(a ; b) & =\left\{\left(d_{1}, d_{2}\right):\left(\exists d_{3} \in D\right)\left(d_{1}, d_{3}\right) \in h(a) \wedge\left(d_{3}, d_{2}\right) \in h(b)\right\}
\end{aligned}
$$

Presented by I. Sain.

Received July 2, 2004; accepted in final form February 22, 2007.

2000 Mathematics Subject Classification: Primary: 03G10, 03G15; Secondary: 06A12, 06F05.

Key words and phrases: algebraic logic, representation, non-finite axiomatisability, semilattice, ordered monoid, reduct. 


\begin{tabular}{lll} 
Signature & Finitely Axiomatisable? & Citation \\
\hline$\{\cdot, ;\}$ & yes & {$[7]$} \\
$\{+, ;\}$ & no & {$[1]$} \\
$\{;,-\}$ & no & {$[5]$} \\
$S \supseteq\{\cdot,+, ;\}$ & no & {$[2$, Theorem 4$]$} \\
$S \supseteq\{\cdot,-, ;\}$ & no & {$[12,8]$} \\
$\left\{\cdot, 1^{\prime}, ;\right\}$ & no & Theorem 2.2
\end{tabular}

Figure 1. Finite Axiomatisability of Representation Classes.

whenever $1^{\prime}, \smile, ; \in S$. An algebra in a signature $S$ is representable iff it belongs to $R(S)$.

So the idea is to remove some of the operations for relation algebras and study the behaviour of the corresponding representation class. For any signature $S \subseteq$ $\left\{0,1,+, \cdot,-, 1^{\prime}, \smile, ;\right\}$ it is trivial that $R(S)$ is closed under isomorphic copies and subalgebras. It is easy to check that $R(S)$ is closed under direct products (just take the disjoint union of the representations of the component algebras). It can also be shown that $R(S)$ is a pseudo-elementary class which is therefore closed under ultraproducts. It follows that $R(S)$ is a quasi-variety, in all cases.

The main focus of this paper is to investigate whether $R(S)$ can be defined by a finite number of axioms, for certain signatures $S$. Thus, if we restrict to just the boolean operations then a representation is just a field of sets in which the boolean operations are interpreted as set-theoretic union and complement. By Stone's Theorem, the axioms for boolean algebra define this representation class. But this may be going too far; we cannot express very much about binary relations in the signature of boolean algebra. So we want to remove as few operations as possible from RA and see if we can achieve better logical and computational behaviour.

The case where we include all the boolean operations and see how many of the other RA operations can be included, while preserving finite axiomatisability, is solved. In the presence of the boolean operations, composition is not finitely axiomatizable, while converse and the identity constant are, see [4]. Thus, a signature between BA and RA has a finitely axiomatisable representation class iff the signature does not contain composition.

But composition is the most interesting of the extra RA-operations. So researchers have also studied the case where we take out or weaken some of the boolean operations and see if we can include composition and perhaps other nonboolean operations, subject to finite axiomatisability. Figure 1 summarises some of the main results. See also [16] for a survey of recent results in this field. 
Our main interest in this paper is algebras in the signature $\left\{\cdot, 1^{\prime}, ;\right\}$ called semilattice-ordered monoids. We do not consider signatures containing negation, so our signatures are positive, but note that with negation and either $\cdot$ or + in the signature, the signature is capable of expressing all the boolean operations, a case we have already dealt with.

\section{Ordered monoids and semilattice-ordered monoids}

In this paper we study two related signatures very close to the border of finite axiomatisability. They are ordered monoids and semilattice-ordered monoids, and their signatures are $\left\{\leq, 1^{\prime}, ;\right\}$ and $\left\{\cdot, 1^{\prime}, ;\right\}$, respectively. It is easy to check (cf. [7]) that every proper subsignature of either of these yields a finitely axiomatisable representation class (where $\leq$ is represented as $\subseteq$ ).

The signature $\left\{\cdot, 1^{\prime}, ;\right\}$ has been the object of some interest (the colloquial term for this language is the "Jerry Fragment", named after Jerry Seligman who studied it because of its connection to channel algebras from situation theory). The problem of whether this signature is finitely axiomatisable was raised by B. Schein, see [16, question 5.1]. The main result in the current paper is that the representation class $R\left(\left\{\cdot, 1^{\prime}, ;\right\}\right)$ is not finitely axiomatisable (Theorem 2.2).

\section{Definition 2.1.}

semi

(1) A semilattice-ordered monoid (SOM) is an algebra in the signature $\left(\cdot, 1^{\prime}, ;\right)$ satisfying

- defines a (lower) semilattice,

- $1^{\prime}$ is an identity for ; i.e., $1^{\prime} ; x=x ; 1^{\prime}=x$,

- ; is associative,

- ; is monotonic with respect to $\leq$, i.e., $x \leq y$ implies $x ; z \leq y ; z$ and $z ; x \leq z ; y$, where $a \leq b$ abbreviates $a \cdot b=a$.

(2) A representation of an algebra $\mathcal{A}$ of this type is a map $h: \mathcal{A} \rightarrow \wp(D \times D)$, for some base set $D$, such that

- $h(a)=h(b) \Longleftrightarrow a=b$,

- $h(a \cdot b)=h(a) \cap h(b)$,

- $h\left(1^{\prime}\right)=\{(d, d): d \in D\}$,

- $h(a ; b)=h(a) \mid h(b)=\{(e, d) \in D \times D:(\exists f \in D)(e, f) \in h(a),(f, d) \in$ $h(b)\}$.

We write ' $\mathcal{A} \in \mathrm{SOM}$ ' or ' $\mathcal{A}$ is a SOM' to mean ' $\mathcal{A}$ is a semilattice-ordered monoid'. Not every semilattice-ordered monoid is representable, as we will see. The question is: can you add finitely many axioms to those defining a semilattice-ordered monoid 
so as to exactly define the representable semilattice-ordered monoids? The answer is negative:

Theorem 2.2. There is no finite set of first-oder axioms in the signature $\left\{\cdot, 1^{\prime}, ;\right\}$ defining the class of representable semilattice-ordered monoids.

This is our main result; the proof is delayed until section 6 .

Corollary 2.3. $R\left(\left\{\cdot, 1^{\prime}, ;\right\}\right)$ is not a variety.

Proof. The equational theory of $R\left(\left\{\cdot, 1^{\prime}, ;\right\}\right)$ is finitely axiomatisable [6]. Then, assuming that $R\left(\left\{\cdot, 1^{\prime}, ;\right\}\right)$ was a variety, there would be a first-order sentence $\varphi$ axiomatising it. By Theorem 2.2, for every finite set $\Psi$ of equations valid in $R\left(\left\{\cdot, 1^{\prime}, ;\right\}\right), \Psi \cup\{\neg \varphi\}$ would be satisfiable. Then, by compactness, $\Phi \cup\{\neg \varphi\}$ would be satisfiable, where $\Phi$ is the set of equations valid in $R\left(\left\{\cdot, 1^{\prime}, ;\right\}\right)$. Since $\{\varphi\}$ and $\Phi$ have the same models, this is a contradiction.

Next we define ordered monoids that are closely related to SOM.

\section{Definition 2.4.}

(1) An ordered monoid $\mathcal{A}=\left(A, \leq, 1^{\prime}, ;\right)$ is a structure where

- $\leq$ is a reflexive partial order on $A$,

- ; is an associative binary operation over $A$,

- $1^{\prime} \in A$ is the identity for ;,

- if $a \leq b$, then $a ; c \leq b ; c$ and $c ; a \leq c ; b$, for any $a, b, c \in A$.

(2) Write $a<b$ if $a \leq b$ but $b \not \leq a$. Clearly $<$ is a strict partial order.

(3) An ordered monoid representation is an injection $h: A \rightarrow \wp(D \times D)$ (for some base set $D$ ) such that

(a) $a \leq b \Longleftrightarrow h(a) \subseteq h(b)$,

(b) $h\left(1^{\prime}\right)=\{(d, d): d \in D\}$,

(c) $h(a ; b)=h(a) \mid h(b)$.

(4) If $h$ is an ordered monoid representation of $\mathcal{A}$ satisfying

(a) for all $x, y \in D$, there is $a \in A$ such that, for all $a^{\prime} \in A$, we have $(x, y) \in$ $h\left(a^{\prime}\right) \Leftrightarrow a \leq a^{\prime}$, and

(b) for all $a \in A$, there is $(x, y) \in h(a)$ such that, for all, $b \in A$, we have $(x, y) \in h(b) \Leftrightarrow a \leq b$,

then we call $h$ a principal ordered monoid representation of $\mathcal{A}$.

Henceforth we will identify an algebra with its domain, so we may write $1^{\prime} \in \mathcal{A}$ instead of $1^{\prime} \in A$, above.

The meet operation '.' defines a partial order in a SOM as follows: $a \leq b \Longleftrightarrow$ $a \cdot b=a$. Thus, from an arbitrary SOM we can define an ordered monoid. The converse is false: a partial order $\leq$ may not define a semilattice, because a pair of 
elements might not have any lower bound and, even if they do, there may not be a unique infimum. Nevertheless, there is a Priestley kind of duality between ordered monoids and SOMs.

Let $\mathcal{A}$ be an ordered monoid. A downset $L$ is a subset of (the domain of) $\mathcal{A}$ such that if $a \in L$ and $b \leq a$ then $b \in L$. For $a \in \mathcal{A}$ the set $a \downarrow={ }_{\operatorname{def}}\{b \in \mathcal{A}: b \leq a\}$ is called a principal downset. We also write $S \downarrow={ }_{\operatorname{def}}\{a \in \mathcal{A}:(\exists s \in S) a \leq s\}$ for any subset $S$ of the domain of $\mathcal{A}$.

Definition 2.5. Let $\mathcal{A}$ be an ordered monoid. We define a SOM-type algebra $\mathcal{A}^{\prime}$ from $\mathcal{A}$ as follows. The domain of $\mathcal{A}^{\prime}$ consists of all downsets of $\mathcal{A}$. The meet operation is defined by intersection: $L \cdot L^{\prime}=_{\text {def }} L \cap L^{\prime}$. It is clear that $L \cdot L^{\prime}$ is a downset and that defines a semilattice of downsets. The identity of $\mathcal{A}^{\prime}$ is $\left(1^{\prime}\right) \downarrow$, and composition is defined by $L ; L^{\prime}=_{\text {def }}\left\{l ; l^{\prime}: l \in L, l^{\prime} \in L^{\prime}\right\} \downarrow$ for any downsets $L, L^{\prime}$.

It is easy to check that this definition of composition of downsets is associative and that $\left(1^{\prime}\right) \downarrow$ acts as the identity over downsets. Trivially, composition is monotonic with respect to set inclusion. Hence,

Lemma 2.6. If $\mathcal{A}$ is an ordered monoid, then $\mathcal{A}^{\prime}$ is a SOM.

Furthermore, for all $a, b \in \mathcal{A}$ we have

$$
(a ; b) \downarrow=a \downarrow ; b \downarrow
$$

We define a theory $\Sigma$ in a two-sorted language. The intended model of this theory will consist of a pair $\left(\mathcal{A}, \mathcal{A}^{\prime}\right)$, where $\mathcal{A}^{\prime}$ is the SOM defined above. A model for $\Sigma$ will be of the form $(A, B)$. Elements of the first sort $(A)$ will be of the type of ordered monoids and elements of the second sort will be of the type of SOMs. The signature of $\Sigma$ includes, as well as the signature of ordered monoids for the first sort and the signature of SOMs for the second sort, a binary predicate $\varepsilon$ whose first argument is of sort one and whose second argument is of sort two. We define $\Sigma$ by first giving an informal description of a formula, then the formal definition.

Definition 2.7. Let $\Sigma$ be the following set of first-oder formulas.

(1) ' $A$ is an ordered monoid' (see Definition 2.4).

(2) 'The elements of $B$ correspond to downsets of $A$ ':

$$
(\forall b \in B)\left(\forall a, a^{\prime} \in A\right)\left(a \varepsilon b \wedge a^{\prime} \leq a \rightarrow a^{\prime} \varepsilon b\right) .
$$

(3) ' $A$ is dense in $B$ ':

$$
\left(\forall b, b^{\prime} \in B\right)\left(b \cdot b^{\prime} \neq b \leftrightarrow(\exists a \in A) a \varepsilon b \wedge a \notin b^{\prime}\right) .
$$

(4) 'The meet operation of $B$ corresponds to intersection of downsets over $A$ ':

$$
\left(\forall b, b^{\prime} \in B\right)(\forall a \in A)\left(a \varepsilon b \cdot b^{\prime} \leftrightarrow a \varepsilon b \wedge a \varepsilon b^{\prime}\right) .
$$


(5) 'The identity of $A$ corresponds to the identity of $B$ ':

$$
(\forall a \in A)\left(a \leq 1_{A}^{\prime} \leftrightarrow a \varepsilon 1_{B}^{\prime}\right) .
$$

(6) 'Composition in $A$ corresponds to composition in $B$ ':

$$
\left(\forall b_{1}, b_{2} \in B\right)(\forall a \in A)\left(a \varepsilon b_{1} ; b_{2} \leftrightarrow\left(\exists a_{1}, a_{2} \in A\right) a \leq a_{1} ; a_{2} \wedge a_{1} \varepsilon b_{1} \wedge a_{2} \varepsilon b_{2}\right) .
$$

Lemma 2.8. Let $\mathcal{A}$ be an ordered monoid. The pair $\left(\mathcal{A}, \mathcal{A}^{\prime}\right)$ is a model of $\Sigma$.

Proof. Just interpret $\varepsilon$ as membership.

Lemma 2.9. Let $\mathcal{A}$ be an ordered monoid with a principal representation and let $\mathcal{M}=(\mathcal{A}, \mathcal{B})$ be a model of the theory $\Sigma$ defined above. Then $\mathcal{B}$ is a representable SOM.

Proof. Let $f$ be a principal ordered monoid representation of $\mathcal{A}$ over the base set $D$. Define $g: \mathcal{B} \rightarrow \wp(D \times D)$ by $g(b)=\bigcup\{f(a): \mathcal{M} \models a \varepsilon b\}$. We claim that $g$ is a SOM representation.

Let $b, b^{\prime} \in \mathcal{B}$ and let $b \neq b^{\prime}$, wlog $b \not \leq b^{\prime}$. By Definition 2.7(3) there is $a \in \mathcal{A}$ such that $\mathcal{M} \models a \varepsilon b \wedge a \notin b^{\prime}$. By the definition of principal ordered monoid representation (Definition 2.4(4b)) there is $(x, y)$ such that $(x, y) \in f\left(a^{\prime}\right) \Leftrightarrow a^{\prime} \geq a$. Since $\mathcal{M} \models$ $a \varepsilon b$, we have $(x, y) \in f(a) \subseteq g(b)$. Also $(x, y) \in g\left(b^{\prime}\right)=\bigcup\left\{f\left(a^{\prime}\right): a^{\prime} \varepsilon b^{\prime}\right\}$ is impossible; indeed $a \notin b^{\prime}$, so by Definition $2.7(2)$ we have $(x, y) \in g(b) \backslash g\left(b^{\prime}\right)$. Hence $g$ is $1-1$.

Next observe that $g\left(b \cdot b^{\prime}\right)=g(b) \cap g\left(b^{\prime}\right)$ by Definition 2.7(4) and Definition 2.4(4a), and $g\left(1_{\mathcal{B}}^{\prime}\right)=f\left(1_{\mathcal{A}}^{\prime}\right)=\{(d, d): d \in D\}$ by Definition 2.7(5). Finally, $g\left(b_{1} ; b_{2}\right)=$ $\bigcup\left\{f(a): \mathcal{M} \models a \varepsilon b_{1} ; b_{2}\right\}=\bigcup\left\{f\left(a_{1} ; a_{2}\right): \mathcal{M} \models a_{1} \varepsilon b_{1} \wedge a_{2} \varepsilon b_{2}\right\}$ by Definition 2.7(6), and the latter is equal to

$$
\bigcup\left\{f\left(a_{1}\right): \mathcal{M} \models a_{1} \varepsilon b_{1}\right\}\left|\bigcup\left\{f\left(a_{2}\right): \mathcal{M} \models a_{2} \varepsilon b_{2}\right\}=g\left(b_{1}\right)\right| g\left(b_{2}\right) .
$$

\section{Characterising representability}

Next we define networks and a representation game using networks.

Definition 3.1. A network $N$ over an ordered monoid $\mathcal{A}$ is a partial map $N: D \times$ $D \rightarrow \mathcal{A}$ for some set of nodes $D$. We say that $N$ is consistent if, for all $d, e, f \in D$, Refl: $N$ is defined on $(d, d)$;

Trans: if $N$ is defined on $(d, e)$ and on $(e, f)$, then it is defined on $(d, f)$;

$I d: N(d, e) \leq 1^{\prime}$ iff $d=e$;

Comp: $N(d, f) \leq N(d, e) ; N(e, f)$.

We denote the set of nodes $D$ by $\operatorname{nodes}(N)$.

We say that $M$ extends $N$, and we write $M \supseteq N$, if $\operatorname{nodes}(M) \supseteq \operatorname{nodes}(N)$ and $M \uparrow_{\operatorname{nodes}(N) \times \operatorname{nodes}(N)}=N$. Given a sequence of (consistent) networks $N_{0} \subseteq$ 
$N_{1} \subseteq \ldots$, we define the limit of the sequence $\bigcup_{i} N_{i}$ to be the network $N$ with $\operatorname{nodes}(N)=\bigcup_{i} \operatorname{nodes}\left(N_{i}\right)$ and labelling $N(x, y)=N_{i}(x, y)$ for any $i$ such that $N_{i}(x, y)$ is defined (since the $N_{i}$ s are nested, this is well defined) and if there is no such $i$ then $N(x, y)$ is undefined. Clearly this is also a (consistent) network. If $N_{i}: i \in I$ are all (consistent) networks and $i \neq j \rightarrow \operatorname{nodes}\left(N_{i}\right) \cap \operatorname{nodes}\left(N_{j}\right)=\emptyset$, then define $N=\bigcup_{i \in I} N_{i}$ to be the network such that $\operatorname{nodes}(N)=\bigcup_{i \in I} \operatorname{nodes}\left(N_{i}\right)$ and $N(x, y)=N_{i}(x, y)$ if $x, y \in \operatorname{nodes}\left(N_{i}\right)$ but $N(x, y)$ is undefined if there is no such $i$. Again, this is a (consistent) network.

Next we define a representation game.

Definition 3.2. The game $G(\mathcal{A})$ on $\mathcal{A}$ has two players $\forall$ (universal) and $\exists$ (existential). A play of the game $G(\mathcal{A})$ consists of a countably infinite sequence of networks $N_{0} \subseteq N_{1} \subseteq \cdots$. In round zero, $\forall$ picks any $\alpha \in \mathcal{A}$. $\exists$ must respond with a consistent network $N_{0}$ containing nodes $x, y$ such that $N_{0}(x, y)=\alpha$. In a later round (round $t+1$ ) suppose $N_{t}$ has just been played. $\forall$ picks nodes $x, y \in N_{t}$ such that $N_{t}$ is defined on $(x, y)$, and he picks any $\alpha, \beta \in \mathcal{A}$ such that $\alpha ; \beta \geq N_{t}(x, y)$. We denote this move as $\left(N_{t}, x, y, \alpha, \beta\right) . \exists$ must respond with a consistent network $N_{t+1}$ extending $N_{t}$ containing a node $z$ where $N_{t+1}(x, z) \leq \alpha$ and $N_{t+1}(z, y) \leq \beta$. If $\exists$ fails to provide the required network in any round, she loses the game. If she succeeds in every round, she wins.

For $n \in \mathbb{N}$ the game $G_{n}(\mathcal{A})$ is similar, but the game ends after round $n$. A play of this game is $N_{0} \subseteq N_{1} \subseteq \cdots \subseteq N_{n}$. If in any of these rounds $\exists$ fails to respond with a suitable network she loses, otherwise she wins.

Proposition 3.3 ([11]). Let $\mathcal{A}$ be an ordered monoid.

(1) If $\mathcal{A}$ has a principal representation, then $\exists$ has a winning strategy in $G(\mathcal{A})$.

(2) If $\mathcal{A}$ is countable and $\exists$ has a winning strategy in $G(\mathcal{A})$, then $\mathcal{A}$ has a principal representation.

(3) If $\exists$ has a winning strategy in $G_{n}(\mathcal{A})$ for all $n \in \mathbb{N}$, then $\mathcal{A}$ is elementarily equivalent to a countable algebra $\mathcal{B}$ for which $\exists$ has a winning strategy in $G(\mathcal{B})$.

(4) For all $n \in \mathbb{N}$, there is a first-order formula $\phi_{n}$ in the language of ordered monoids such that, for all ordered monoids $\mathcal{B}$, we have $\mathcal{B} \models \phi_{n}$ if and only if $\exists$ has a winning strategy in $G_{n}(\mathcal{B})$.

Proof. (Sketch.)

(1) Let $h$ be a principal representation of $\mathcal{A}$ with domain $D$. For her winning strategy, $\exists$ maintains a map ' $: \operatorname{nodes}(N) \rightarrow D$, where $N$ is any network played in the game, such that if $N(x, y)$ is defined, then it is the generator of the filter of elements holding on $\left(x^{\prime}, y^{\prime}\right)$ in the representation, i.e., $a \geq N(x, y) \Longleftrightarrow$ $\left(x^{\prime}, y^{\prime}\right) \in h(a)$ for any $a \in \mathcal{A}$. 
(2) Suppose $\mathcal{A}$ is countable and $\exists$ has a winning strategy. In a play of $G(\mathcal{A})$ suppose $\forall$ plays $\alpha$ in the initial round and then plays all possible moves eventually, i.e., if $N_{t}$ occurs in the play and $\beta ; \gamma \geq N_{t}(x, y)$ then at some later round of the game $(s>t), \forall$ plays $\left(N_{s}, x, y, \beta, \gamma\right)$. Since $\mathcal{A}$ is countable, this can be scheduled. Let $\exists$ use her winning strategy in such a game and let $N_{\alpha}$ be the limit of the game. By renaming the nodes, if necessary, we can suppose that $\operatorname{nodes}\left(N_{\alpha}\right) \cap \operatorname{nodes}\left(N_{\alpha^{\prime}}\right)=\emptyset$, whenever $\alpha \neq \alpha^{\prime}$. Now let $N=\bigcup_{\alpha \in \mathcal{A}} N_{\alpha}$. For a principal representation of $\mathcal{A}$ over the domain $\operatorname{nodes}(N)$, let

$$
h(a)=\{(x, y): N(x, y) \text { is defined and } N(x, y) \leq a\} .
$$

(3) See [11, Proposition 10.14(2)].

(4) See [11, Theorem 10.12 and Proposition 10.13].

\section{Non-representability}

Let us return to our main problem whether the class of representable SOM is finitely axiomatizable.

A standard way of proving non-finite axiomatisability is to use an ultraproduct construction to show that the complement of the class of algebras is not closed under ultraproducts. So, for example, the representation class $R\left(\left\{\cdot, 1^{\prime}, \smile, ;\right\}\right)$ was shown to be non-finitely axiomatisable by constructing a sequence of non-representable algebras (so-called rainbow algebras) with a classically representable ultraproduct [12]. The signature for this class is very similar to that of SOM, but our attempts to modify this construction to obtain a non-finite axiomatisability result for SOM have failed. But if we could establish that converse is finitely axiomatisable over $\left\{\cdot, 1^{\prime}, ;\right\}$ (as is the case where all boolean operations are present [4]), then nonfinite axiomatisability for representable SOM would follow. Alas this is not case, as Corollary 4.2 shows.

Theorem 4.1. Let $\mathcal{A}$ be a SOM, and assume that $\mathcal{A}$ has a minimal (with respect to the ordering $\leq$ ) zero element 0 such that

(1) $0 ; a=a ; 0=0$,

(2) $1^{\prime}$ is a minimal non-zero element,

(3) $a ; b \leq 1^{\prime} \rightarrow\left(a \leq 1^{\prime} \vee b \leq 1^{\prime}\right)$.

Then $\mathcal{A}$ is representable.

Observe that the last two conditions are not sound over representable SOMs.

Proof. Suppose $\mathcal{A}$ is countable and satisfies the conditions. We will define a winning strategy for $\exists$ in a modified version $G^{\prime}(\mathcal{A})$ of the game $G(\mathcal{A})$ (see Definitions 3.1, 
3.2). In the initial round of $G^{\prime}(\mathcal{A}), \forall$ can pick any element of $\mathcal{A} \backslash\{0\}$ (so the modification is that he is not allowed to pick zero). In each round $\exists$ must play a consistent network $N$ satisfying:

Zero: $N(m, n) \neq 0$,

for any nodes $m, n \in \operatorname{nodes}(N)$. In all other respects the game $G^{\prime}(\mathcal{A})$ is the same as $G(\mathcal{A})$.

In the initial round of $G^{\prime}(\mathcal{A}), \forall$ chooses any $a \in \mathcal{A} \backslash\{0\}$. If $a=1^{\prime}$ then $\exists$ plays a network $N_{0}$ with just one node $n$ and labelling $N_{0}(n, n)=1^{\prime}$. Otherwise $\left(a \not \leq 1^{\prime}\right), \exists$ lets $N_{0}$ have two nodes $m, n$ and labelling $N_{0}(m, m)=N_{0}(n, n)=1^{\prime}, N_{0}(m, n)=a$ (the edge $(n, m)$ is unlabelled).

In a later round, suppose the network $N$ has just been played. $\forall$ chooses nodes $m, n \in N$ and elements $a, b \in \mathcal{A}$ such that $a ; b \geq N(m, n)$. Since $N(m, n) \neq 0$ it follows that $a \neq 0$ and $b \neq 0 . \exists$ must define a network $M \supseteq N$ containing a node $l$ such that $M(m, l) \leq a$ and $M(l, n) \leq b$. If $a=1^{\prime}$ she can let $M=N$ and let $l=m$, and if $b=1^{\prime}$ she lets $M=N$ and $l=n$. Assuming that neither $a \leq 1^{\prime}$ nor $b \leq 1^{\prime}$, she lets $M$ have exactly one new node $l$ not occurring as a node of $N$ and defines labels of edges incident with $l$ by

$$
\begin{aligned}
M(l, l) & =1^{\prime}, \\
M(u, l) & =N(u, m) ; a, \\
M(l, u) & =b ; N(n, u),
\end{aligned}
$$

where $u \in N$ is arbitrary. (We use the convention that $N(u, m) ; a$ is undefined if $N(u, m)$ is undefined.) Let us check that $M$ is a consistent network. Network conditions Refl and Trans are easy to check. For network condition Comp take any $u, v \in \operatorname{nodes}(N)$. We have

$$
\begin{aligned}
M(u, l) ; M(l, v) & =(N(u, m) ; a) ;(b ; N(n, v)) \\
& \geq N(u, m) ; N(m, n) ; N(n, v) \\
& \geq N(u, v)=M(u, v), \\
M(u, v) ; M(v, l) & =N(u, v) ;(N(v, m) ; a) \\
& \geq N(u, m) ; a=M(u, l),
\end{aligned}
$$

and similarly $M(l, u) ; M(u, v) \geq M(l, v)$. To show that the remaining conditions Id and Zero for networks are true in $M$, we will show that $M(u, l), M(l, u) \not \leq 1^{\prime}$ for $u \in$ $\operatorname{nodes}(N)$. So, for contradiction, suppose $M(u, l) \leq 1^{\prime}$ for some $u \in \operatorname{nodes}(N)$. Then $M(u, l)=N(u, m) ; a \leq 1^{\prime}$. So, by condition 3 of the theorem, either $N(u, m) \leq 1^{\prime}$ or $a \leq 1^{\prime}$. We are assuming $a \not \leq 1^{\prime}$. If $N(u, m) \leq 1^{\prime}$ then $u=m$, but then $M(u, l)=M(m, l)=a \not \leq 1^{\prime}$. Thus $M(u, l) \leq 1^{\prime}$ is impossible. Similarly, we cannot 
have $M(l, u) \leq 1^{\prime}$ for any $u \in \operatorname{nodes}(N)$. Thus $M$ is a network, whence $\exists$ has a winning strategy for $G^{\prime}(\mathcal{A})$.

Now let $N$ be the limiting network in a play of $G^{\prime}(\mathcal{A})$ where $\exists$ uses her winning strategy and $\forall$ plays all possible moves - i.e., if $N_{i}$ is played at some point and $m, n \in \operatorname{nodes}\left(N_{i}\right)$ and $a ; b \geq N_{i}(m, n)$, then at some point $\forall$ will pick $m, n$ and $a, b$ for his move. Since each network occurring in the play is finite and $\mathcal{A}$ is countable, it is possible to schedule all these moves in the play. As in Proposition 3.3, a representation $h$ of $\mathcal{A}$ with base nodes $(N)$ can now be defined by

$$
h(a)=\{(m, n) \in \operatorname{nodes}(N) \times \operatorname{nodes}(N): N(m, n) \leq a\} .
$$

In fact, this defines a complete representation, where arbitrary infima are preserved.

Finally, if $\mathcal{B}$ is a not necessarily countable SOM satisfying the condition, then let $\mathcal{A} \prec \mathcal{B}$ be a countable elementary subalgebra. Then $\mathcal{A}$ also satisfies the condition and by the above $\mathcal{A}$ is representable. Since the class of representable SOMs is a quasi-variety, it is closed under elementary equivalence, hence $\mathcal{B}$ is also representable.

A generalisation of the theorem to algebras where $1^{\prime}$ need not be minimal can be obtained, but the axioms, definitions and proof are slightly more complicated.

Corollary 4.2. $R\left(\left\{\cdot, 1^{\prime}, \smile, ;\right\}\right)$ is not finitely axiomatisable over $R\left(\left\{\cdot, 1^{\prime}, ;\right\}\right)$.

Proof. Suppose, for contradiction, that $R\left(\left\{\cdot, 1^{\prime}, \smile, ;\right\}\right)$ is defined by an axiomatisation $\Theta$ of $R\left(\left\{\cdot, 1^{\prime}, ;\right\}\right)$ together with a finite set of axioms. Without loss we can suppose that this finite set consists of a single first-order axiom $\phi$. So our supposition is that for any $\left\{\cdot, 1^{\prime}, \smile, ;\right\}$-algebra $\mathcal{B}$ we have $\mathcal{B} \in R\left(\left\{\cdot, 1^{\prime}, \smile, ;\right\}\right) \Leftrightarrow \mathcal{B} \models \Theta \cup\{\phi\}$.

Now let $\mathcal{A}_{n}$ be the rainbow algebra of $[12$, section 3.1]. This is a finite relation algebra. We will slightly abuse notation for the sake of brevity. If $S^{\prime} \subseteq S$ and $\mathcal{A}$ is an $S$-algebra, then by $\mathcal{A} \in R\left(S^{\prime}\right)$ we mean that the $S^{\prime}$-reduct of $\mathcal{A}$ is in $R\left(S^{\prime}\right)$. The identity of $\mathcal{A}_{n}$ is minimal non-zero, for $x, y \in \mathcal{A}_{n}$ we have $x ; y \leq 1^{\prime} \rightarrow\left(x \leq 1^{\prime} \vee y \leq\right.$ $\left.1^{\prime}\right)$, and all the other conditions of Theorem 4.1 hold. Hence, $\mathcal{A}_{n} \in R\left(\left\{\cdot, 1^{\prime}, ;\right\}\right)$. By [12, Lemma 3.1], $\mathcal{A}_{n} \notin R\left(\left\{\cdot, 1^{\prime}, \smile, ;\right\}\right)$. But by [12, Lemma 3.4], for any nonprincipal ultraproduct $\mathcal{A}$ of the $\mathcal{A}_{n}$ s we have $\mathcal{A} \in R\left(\left\{\cdot, 1^{\prime}, \smile, ;\right\}\right)$.

By our assumption, $\mathcal{A} \models \Theta \cup\{\phi\}$. By Loś' theorem, $\mathcal{A}_{n} \models \phi$ for "many" values of $n$. Since $\mathcal{A}_{n} \in R\left(\left\{\cdot, 1^{\prime}, ;\right\}\right)$, we have $\mathcal{A}_{n} \models \Theta$, for all $n$. But then, for "many" $n$, we have $\mathcal{A}_{n} \models \Theta \cup\{\phi\}$ so $\mathcal{A}_{n} \in R\left(\left\{\cdot, 1^{\prime},{ }^{\smile}, ;\right\}\right)$, contradicting [12, Lemma 3.1].

We are about to define a sequence of unrepresentable algebras $\mathcal{A}_{n}: n \geq 1$ with a representable ultraproduct. This will allow us to prove the non-finite axiomatisability of the representation class. We have to admit that the construction of the algebras is a little complex and the proof of representability of the ultraproduct is fearsome. Let us try to explain how we arrived at such a construction. 
When we looked at the signature $\left\{\cdot, 1^{\prime}, ;\right\}$ we first attempted to prove that $R\left(\left\{\cdot, 1^{\prime}, ;\right\}\right)$ was finitely axiomatisable. We wrote down a small number of axioms - mostly quite obvious axioms like monotonicity and associativity, but also some extra axioms like $e, e^{\prime} \leq 1^{\prime} \rightarrow e ; e^{\prime}=e \cdot e^{\prime}$ and $e \leq 1^{\prime} \wedge e ; x \geq y \rightarrow e ; y \geq y$ — and attempted to prove that these axioms were sufficient for representability. Now, if a SOM does not contain any elements $\bar{f}, f \not \leq 1^{\prime}$ such that $\bar{f} ; f \leq 1^{\prime}$ then it is easy to prove that such an algebra satisfying this set of axioms must be representable. You just build an antisymmetric representation of the algebra, step by step; cf. Theorem 4.1. Without such "functional elements", the identity is not playing an important role and the representability of an algebra reduces to its representability for the signature $\{\cdot, ;\}$.

We were even able to prove representability if the algebra contained elements $\bar{f}_{i}, f_{i}: i \in I$ with $\bar{f}_{i} ; f_{i} \leq 1^{\prime}$ or $f_{i} ; \bar{f}_{i} \leq 1^{\prime}$, provided that the following condition holds: if there is an element $b$ with $b \leq s$ for some product $s$ of elements from $\left\{f_{i}, \bar{f}_{i}: i \in I\right\}$, then $b$ is equal to such a product. Very roughly, we could build clusters of points related by invertible functions and relate clusters to each other by antisymmetric relations as in the previous paragraph. But we could not extend our proof of representability to cover the case where elements such as $b$ exist.

The failure of our proof led us to construct an unrepresentable semilatticeordered monoid whose elements included $f, \bar{f}, g, \bar{g}, b$ where $\bar{f} ; f \leq 1^{\prime}, g ; \bar{g} \leq 1^{\prime}$ and $b<f ; g$ but $b$ is not equal to any product of $\{f, \bar{f}, g, \bar{g}\}$. It also includes elements $a, c$ and satisfies $a ; b \geq c$. Our example is essentially $\mathcal{A}_{1}^{\prime}$ of Definition 4.9 , below. Because we have $\bar{f} ; f \leq 1^{\prime}$ and $f ; \bar{f} \geq 1^{\prime}$, then if a representation of the algebra were to exist, whenever an edge $(x, y)$ belonged to the representation of $f$ it would follow that $(y, x)$ belonged to the representation of $\bar{f}$ and vice versa, and a similar property would hold for $g, \bar{g}$. We can define a sort of converse operator on products of elements from $\{f, \bar{f}, g, \bar{g}\}$ by reversing the product and adding/removing the overline. Products of elements from $\{f, \bar{f}, g, \bar{g}\}$ are reversible in the sense that whenever an edge belonged to the representation of such a product, the reverse edge must belong to the representation of the converse. Part of the problem, in attempting to represent the example we constructed, seems to be that $b$ is also reversible - if $(x, y)$ belonged to the representation of $b$ then $(y, x)$ would belong to the representation of $\bar{g} ; \bar{f}$ - but $\bar{g} ; \bar{f}$ is not a true converse to $b$.

Straight after this discovery we switched from trying to find a finite number of axioms to define $R\left(\left\{\cdot, 1^{\prime}, ;\right\}\right)$ to proving that no such finite set of axioms exists. Our aim was to define an unrepresentable ordered monoid $\mathcal{A}_{n}$ for each finite $n>0$, and show that $\mathcal{A}_{n}^{\prime}$ is an unrepresentable SOM (Definition 2.5 above explains how the SOM $\mathcal{A}_{n}^{\prime}$ is constructed from the ordered monoid $\left.\mathcal{A}_{n}\right)$. Later we will show that an algebra which is elementarily equivalent to a non-principal ultraproduct of the 
$\mathcal{A}_{n} \mathrm{~s}$ has a principal representation, and then we will be able to deduce our main result - the class of representable SOMs is not finitely axiomatisable.

So we tried to devise an unrepresentable SOM, but we wanted to define it so that the assumption that the SOM is representable only led to a contradiction if more than $n$ points in a claimed representation were considered, for $n \in \mathbb{N}$. This helped us prove the representability of a non-principal ultraproduct of these unrepresentable SOMs.

In order to do this we had to generalise our construction so that more and more points had to be considered, in a hypothetical representation of the algebra, to obtain a contradiction. This generalisation is given below. Here we have $b \leq$ $f ; g ; f ; \cdots ; g$ (a product of $2 n$ elements) and we have replaced $c$ by $k_{f ; g ; f ; \cdots ; g}$. Additional elements have also been introduced, for reasons we discuss later.

The construction is based on strings over an alphabet including $\bar{f}, f, \bar{g}, g, a, b$ and some other characters, and composition is essentially determined by concatenation. This is an easy way of ensuring the associativity of our algebras. The alphabet also includes characters $k_{s}$. Without these extra characters the algebras could be proved nonrepresentable by considering only five points in a claimed representation, as we will explain in more detail below (see remark 4.12).

4.1. Strings and normal forms. For any set $A$, we let $A^{*}$ denote the set of all strings over $A$. For any string $s,|s|$ denotes the length of the string, and for $k \in \mathbb{N}, s^{k}$ denotes the string obtained by concatenating $k$ copies of $s$ together; by convention we let $s^{0}$ denote $\Lambda$, the empty string. Let $F=\{f, \bar{f}, g, \bar{g}\}$. For any string $s \in F^{*}, \bar{s}$ is the string obtained from $s$ by replacing all occurrences of $f, \bar{f}, g, \bar{g}$ respectively by $\bar{f}, f, \bar{g}, g$, and reversing the order of the string.

Fix $n \geq 1$. Let $S_{n}$ be the set of strings over the alphabet

$$
\Sigma_{n}=\{a, b, f, \bar{f}, g, \bar{g}\} \cup\left\{k_{s}: s \text { is an initial segment (i.s.) of }(f g)^{n}\right\} .
$$

So our alphabet has $6+(2 n+1)$ characters. When reading the following definition, bear in mind that the empty string $\Lambda$ will play the role of the identity in our algebras.

\section{Definition 4.3.}

- We define a binary relation $\prec$ over $S_{n}$.

$$
\begin{aligned}
& \Lambda \prec f \bar{f} \\
& \Lambda \prec \bar{g} g \\
& \Lambda \prec \bar{f} f \prec \Lambda \\
& \Lambda \prec g \bar{g} \prec \Lambda
\end{aligned}
$$




$$
\begin{aligned}
b & \prec(f g)^{n} \\
(f g)^{n-1} f & \prec b \bar{g} \\
g(f g)^{n-1} & \prec \bar{f} b \\
k_{\Lambda} & \prec a \\
k_{(f g)^{n}} & \prec a b \\
k_{\Lambda} b & \prec k_{(f g)^{n}} \bar{g} g \\
k_{s \phi} & \prec k_{s} \phi \quad \phi \in\{f, g\}, s \phi \text { is an i.s. of }(f g)^{n} \\
k_{s} & \prec k_{s \phi} \bar{\phi} \quad \phi \in\{f, g\}, s \phi \text { is an i.s. of }(f g)^{n}
\end{aligned}
$$

- If $\tau$ is one of the reduction listed above, say $\tau=\left(t^{\prime} \succ t\right)$, then we may write $s t^{\prime} u \stackrel{\tau}{\rightarrow} s t u$ and we say that $\tau$ is a reduction from $s t^{\prime} u$ to $s t u$.

- We define binary relations $\leq_{k}$ over $S_{n}$, for $k \in \mathbb{N}$.

$\leq_{0}$ is defined to be equality.

$\leq_{1}={ }_{\operatorname{def}}\left\{\left(s t u, s t^{\prime} u\right): t \prec t^{\prime}, s, t, u \in S_{n}\right\}$. $\leq_{k+1}={ }_{\text {def }} \leq_{k} \mid \leq_{1}$.

Now define $\leq=\bigcup\left\{\leq_{k}: k \in \mathbb{N}\right\}$, the reflexive transitive closure of $\leq_{1}$.

- We write $s \equiv t$ iff $s \leq t$ and $t \leq s$, and we write $s<t$ if $s \leq t$ but $t \not \leq s$.

Care is needed when using the $x \stackrel{\tau}{\rightarrow} y$ notation as $x$ and $\tau$ do not determine $y$ (e.g. let $x=(f g)^{n}(f g)^{n}$ and let $\left.\tau=\left((f g)^{n} \succ b\right)\right)$. Also, $x$ and $y$ do not determine $\tau$ (e.g. let $x=f \bar{f} f, y=f$ then $\tau=(f \bar{f} \succ \Lambda)$ and $\tau=(\bar{f} f \succ \Lambda)$ are both possible).

Lemma 4.4. The following equivalences are true in $\left(S_{n}, \leq\right)$.

$$
\begin{aligned}
b \bar{g} & \equiv(f g)^{n-1} f & & \\
\bar{f} b & \equiv g(f g)^{n-1} & & \\
k_{s} f & \equiv k_{s f} & & \text { sf is an i.s. of }(f g)^{n} \\
k_{t} & \equiv k_{t g} \bar{g} & & \text { tg is an i.s. of }(f g)^{n} \\
k_{(f g)^{n}} \bar{g} & \equiv k_{s} t & & \text { stg }=(f g)^{n}
\end{aligned}
$$

Here are the proofs.

Proof.

$$
\begin{aligned}
b \bar{g} & \geq(f g)^{n-1} f \equiv(f g)^{n-1} f g \bar{g} \geq b \bar{g} \\
\bar{f} b & \geq g(f g)^{n-1} \equiv \bar{f} f g(f g)^{n-1} \geq \bar{f} b \\
k_{s} f & \geq k_{s f} \equiv k_{s f} \bar{f} f \geq k_{s} f
\end{aligned}
$$




$$
\begin{aligned}
k_{t} & \equiv k_{t} g \bar{g} \geq k_{t g} \bar{g} \geq k_{t} \\
k_{(f g)^{n}} \bar{g} & \equiv k_{(f g)^{n}} \bar{g} g \bar{g} \geq k_{\Lambda} b \bar{g} \equiv k_{\Lambda}(f g)^{n-1} f \\
& \geq k_{s} t \geq k_{(f g)^{n-1} f} \equiv k_{(f g)^{n}} \bar{g}
\end{aligned}
$$

Thus, many distinct strings can be equivalent. Next we define a normal form $\operatorname{nf}(x)$ equivalent to a given string $x$, and then show that $\operatorname{nf}(x)$ determines $x$ up to equivalence (Lemma 4.8).

Definition 4.5. Define $\operatorname{nf}(x)$ from $x$ by repeatedly performing one of the replacements below until no more replacements are possible. At any stage, if there is more than one possible replacement that can be performed then (for now) use a fixed but arbitrary order to choose which one to select. See Corollary 4.7 below.

\begin{tabular}{l|l|l|l} 
& Replace & By & Condition \\
\hline 1 & $\bar{f} f$ & $\Lambda$ & \\
2 & $g \bar{g}$ & $\Lambda$ & \\
3 & $b \bar{g}$ & $(f g)^{n-1} f$ & \\
4 & $\bar{f} b$ & $g(f g)^{n-1}$ & \\
5 & $k_{s} t$ & $k_{\Lambda} s t$ & $s t=(f g)^{n-1} f, s \neq \Lambda$ \\
6 & $k_{s f}$ & $k_{s} f$ & $s f$ is an i.s. of $(f g)^{n}$ \\
7 & $k_{s f g} \bar{g}$ & $k_{s} f$ & $s f$ is an i.s. of $(f g)^{n}$
\end{tabular}

Lemma 4.6 (Commutating replacements). Let $x, y, z \in S_{n}$ and suppose $\sigma, \tau$ are replacements listed in Definition 4.5 such that $x \stackrel{\sigma}{\rightarrow} y$ and $x \stackrel{\tau}{\rightarrow} z$. Then there are two replacements $\sigma^{\prime}, \tau^{\prime}$ and $w \in S_{n}$ such that $y \stackrel{\tau^{\prime}}{\rightarrow} w$ and $z \stackrel{\sigma^{\prime}}{\rightarrow} w$ where $\sigma^{\prime}, \tau^{\prime}$ are either the identity replacement or belong to the list of replacements in Definition 4.5 above.

Proof. Let $\sigma=\left(s \succ s^{\prime}\right), \tau=\left(t \succ t^{\prime}\right)$. So $x$ contains substrings $s, t$ (possibly $x$ contains more than one substring $s$, but a unique occurrence of $s$ is replaced using $\sigma)$. If these two substrings are disjoint in $x$ then clearly there is $w$ such that $y \stackrel{\tau}{\rightarrow} w$ and $z \stackrel{\sigma}{\rightarrow} w$, so we can take $\tau^{\prime}=\tau$ and $\sigma^{\prime}=\sigma$.

If $y=z$ we can let $w=y$ and both of $\sigma^{\prime}$ and $\tau^{\prime}$ are the identity replacement.

That leaves the case where $s, t$ are overlapping but not equal substrings of $x$. There are just two possibilities. It could be that $s=b \bar{g}$ and $t=\bar{f} b$ (or the other way round). In this case, $x=x_{1} \bar{f} b \bar{g} x_{2}$ (for some $\left.x_{1}, x_{2}\right), y=x_{1} \bar{f}(f g)^{n-1} f x_{2}$ and $z=x_{1} g(f g)^{n-1} \bar{g} x_{2}$. The required $w$ is $x_{1} g(f g)^{n-2} f x_{2}$. The other case is where $s=k_{u f}$ and $t=k_{u f} v$, where $u f v=(f g)^{n-1} f$, (or the other way round), so $x=x_{1} k_{u f} v x_{2}, y=x_{1} k_{u} f v x_{2}$ and $z=x_{1} k_{\Lambda}(f g)^{n-1} f x_{2}$. In this case we can let $w=z$. 
Hence,

Corollary 4.7. When computing the normal form $\operatorname{nf}(x)$ of any $x \in S_{n}$ (Definition 4.5) it does not matter what order is chosen to perform the replacements. That is, every $x \in S_{n}$ has an equivalent unique normal form $\operatorname{nf}(x)$ not dependent on the chosen order for the replacements.

Furthermore,

Lemma 4.8. Let $x, y \in S_{n}$. The following are equivalent.

- $x \equiv y$.

- $\operatorname{nf}(x)=\operatorname{nf}(y)$.

Proof. Clearly $x \equiv \operatorname{nf}(x)$ (use Lemma 4.4) so the second statement implies the first. Conversely, suppose $x \equiv y$. We prove that $\operatorname{nf}(x)=\operatorname{nf}(y)$ by induction on the length $(k)$ of the chain $x \geq_{k} y$. If $k=0$ then $x=y$ so trivially $\operatorname{nf}(x)=\operatorname{nf}(y)$. Assume that $x \equiv y$ and $x \geq_{k} y$ implies $\operatorname{nf}(x)=\operatorname{nf}(y)$. Now suppose $x \geq_{1} x^{\prime} \geq_{k} y$, say $x \stackrel{\tau}{\rightarrow} x^{\prime}$ for some reduction $\tau$ from Definition 4.3 , and $x \equiv y$. Since $x^{\prime} \equiv y$ it follows inductively that $\operatorname{nf}\left(x^{\prime}\right)=\operatorname{nf}(y)$. If $\tau$ is any of the following reductions then, by Corollary 4.7, $\operatorname{nf}(x)=\operatorname{nf}\left(x^{\prime}\right)$ and hence $\operatorname{nf}(x)=\operatorname{nf}(y)$, as required: $\bar{f} f \succ 1^{\prime}$, $1^{\prime} \succ \bar{f} f, g \bar{g} \succ 1^{\prime}, 1^{\prime} \succ g \bar{g}, b \bar{g} \succ(f g)^{n-1} f, \bar{f} b \succ g(f g)^{n-1}, k_{s} f \succ k_{s f}$ or $k_{t g} \bar{g} \succ k_{t}$, where $s f, t g$ are arbitrary initial segments of $(f g)^{n}$. Also, if $s \phi t=(f g)^{n-1} f$ and if $\tau$ satisfies $k_{s} \phi t \stackrel{\tau}{\rightarrow} k_{s \phi} t$, then by the fifth replacement listed in Definition 4.5 and by Corollary 4.7 we have $\operatorname{nf}(x)=\operatorname{nf}\left(x^{\prime}\right)$ so, again, $\operatorname{nf}(x)=\operatorname{nf}(y)$. That leaves the following possibilities for $\tau$ : (i) $(f g)^{n} \succ b$, (ii) $a \succ k_{\Lambda}$, (iii) $a b \succ k_{(f g)^{n}}$, (iv) $k_{(f g)^{n}} \bar{g} g \succ k_{\Lambda} b$, (v) $k_{t} g \succ k_{t g}$ or (vi) $k_{s f} \bar{f} \succ k_{s}$. For the first alternative, if there is a string $\gamma \equiv \bar{g}$ immediately to the right of $(f g)^{n}$ or a string $\phi \equiv \bar{f}$ immediately to the left of $(f g)^{n}$, then $\operatorname{nf}(x)=\operatorname{nf}\left(x^{\prime}\right)$; if there is no such string then $x>x^{\prime}$, contrary to assumption. The second and third alternatives are impossible, since they each imply $x \not \equiv x^{\prime}$. For the fourth alternative, if there is a string $\gamma \equiv \bar{g}$ immediately to the right of $k_{(f g)^{n}} \bar{g} g$, then $\operatorname{nf}(x)=\operatorname{nf}\left(x^{\prime}\right)$ as required, else $x \not \equiv x^{\prime}$ contrary to assumption. For the fifth alternative, if there is a string $\gamma$ immediately to the right of $k_{t} g$ with either $\gamma \equiv \bar{g}$ or $t g \gamma \equiv(f g)^{n-1} f$, then $\operatorname{nf}(x)=\operatorname{nf}\left(x^{\prime}\right)$, else $x \not \equiv x^{\prime}$. And for the sixth alternative, if there is a string $\phi \equiv f$ immediately to the right of $k_{s f} \bar{f}$, then $\operatorname{nf}(x)=\operatorname{nf}\left(x^{\prime}\right)$, else $x \not \equiv x^{\prime}$. In each case we either derive a contradiction by showing $x \not \equiv x^{\prime}$ or we deduce that $\operatorname{nf}(x)=\operatorname{nf}(y)$ as required.

Also note that $k_{\Lambda}$ is in normal form. By Definition 4.3, we have $k_{\Lambda} \leq a$ (item $(3))$, and $k_{\Lambda} \leq k_{(f g)^{n}}(\bar{g} \bar{f})^{n}$ (item (5)). 


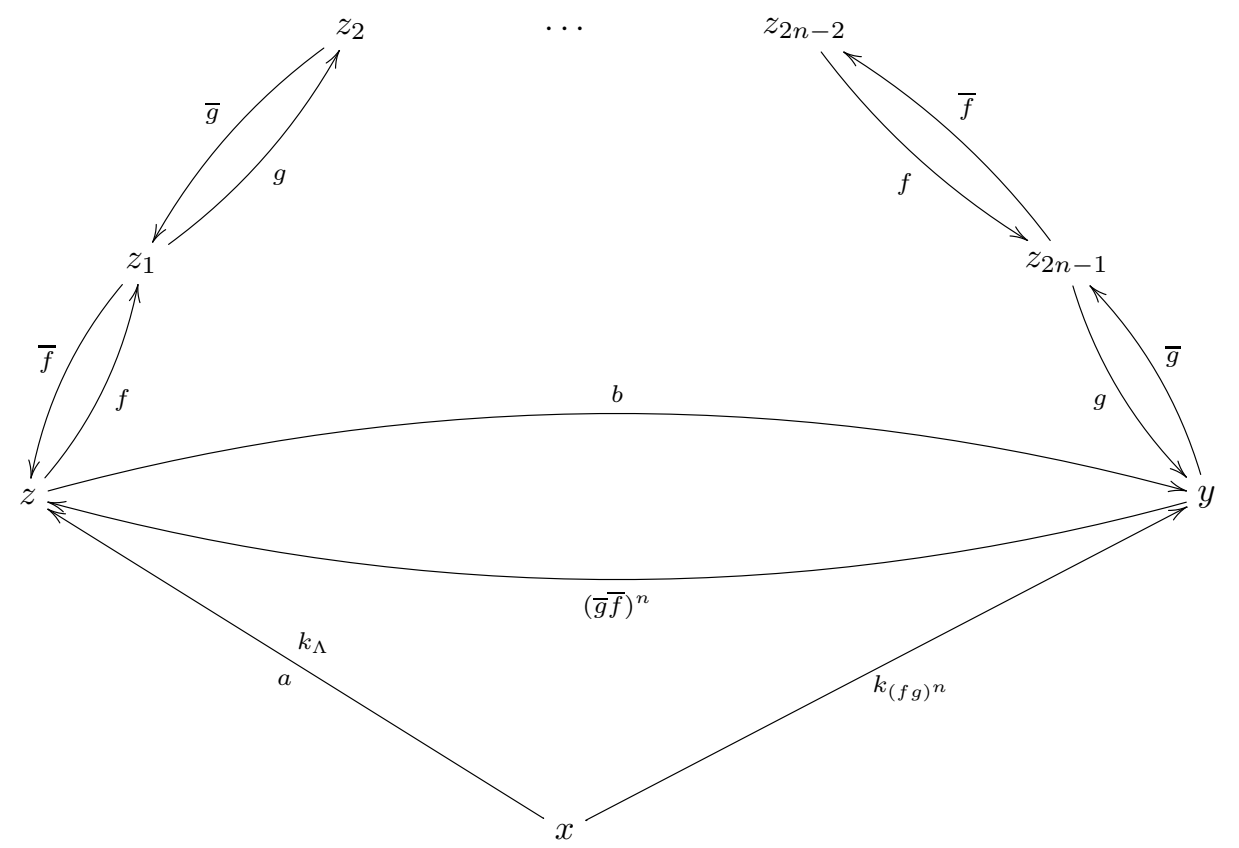

FIGURE 2.

4.2. Non-representable algebras. We are in a position to define non-representable algebras for every $1 \leq n \in \mathbb{N}$.

Definition 4.9. Let $n \geq 1$. Let $A_{n}=\left\{\operatorname{nf}(x): x \in S_{n}\right\}$. For $x, y \in A_{n}$, let $x ; y=\operatorname{nf}(x y)$, i.e., we take the normal form of the concatenation of $x$ and $y$. Let $\mathcal{A}_{n}=\left(A_{n}, \leq, 1^{\prime}, ;\right)$, where $1^{\prime}$ is the empty string $\Lambda$.

Lemma 4.10. For $n \geq 1, \mathcal{A}_{n}$ is an ordered monoid.

Proof. Use Lemma 4.6.

Theorem 4.11. $\mathcal{A}_{n}^{\prime}$ is an unrepresentable SOM.

Proof. By Lemmas 2.6 and 4.10, $\mathcal{A}_{n}^{\prime}$ is a SOM. Suppose for contradiction that there is a SOM representation $h: \mathcal{A}_{n}^{\prime} \rightarrow \wp(D \times D)$ (see figure 2). Note that $\emptyset$ is a downset of $\mathcal{A}_{n}$, so it is the least element of $\mathcal{A}_{n}^{\prime}$. 


$$
\begin{aligned}
\emptyset \neq k_{(f g)^{n} \downarrow} & \Rightarrow(\exists x, y \in D)(x, y) \in h\left(k_{\left.(f g)^{n} \downarrow\right) \backslash h(\emptyset)}\right. \\
k_{(f g)^{n} \downarrow \leq(a ; b) \downarrow} & \Rightarrow(\exists z \in D)(x, z) \in h(a \downarrow) \wedge(z, y) \in h(b \downarrow) \\
b \downarrow \leq(f g)^{n} \downarrow & \Rightarrow\left(\exists z_{1}, \ldots, \exists z_{2 n-1}\right)\left(z, z_{1}\right) \in h(f \downarrow),\left(z_{1}, z_{2}\right) \in h(g \downarrow), \\
& \ldots,\left(z_{2 n-1}, y\right) \in h(g \downarrow) \\
f \downarrow ; \bar{f} \downarrow \geq 1^{\prime} \downarrow & \Rightarrow \exists w((z, w) \in h(f \downarrow) \wedge(w, z) \in \bar{f} \downarrow) \\
& \Rightarrow\left(w, z_{1}\right) \in h((\bar{f} ; f) \downarrow)=h\left(1^{\prime} \downarrow\right) \\
& \Rightarrow w=z_{1},\left(z_{1}, z\right) \in h(\bar{f} \downarrow) \\
\text { Similarly } & \left(z_{3}, z_{2}\right) \in h(\bar{f} \downarrow), \ldots,\left(z_{2 n-1}, z_{2 n-2}\right) \in h(\bar{f} \downarrow) \\
\text { Similarly: } & \left(z_{2}, z_{1}\right) \in h(\bar{g} \downarrow), \ldots,\left(y, z_{2 n-1}\right) \in h(\bar{g} \downarrow) \\
\text { Putting together: } & (y, z) \in h((\bar{g} ; \bar{f} ; \ldots ; \bar{g} ; \bar{f}) \downarrow)=h\left((\bar{g} \bar{f})^{n} \downarrow\right) \\
& \Rightarrow(x, z) \in h\left(\left(k_{(f g)^{n}} ;(\bar{g} \bar{f})^{n}\right) \downarrow\right) \cap h(a \downarrow) \\
& \Rightarrow(x, z) \in h\left(\left(k_{(f g)^{n}} ;(\bar{g} \bar{f})^{n}\right) \downarrow \cdot a \downarrow\right)=h\left(k_{\Lambda} \downarrow\right) \\
& \Rightarrow(x, y) \in h\left(\left(k_{\Lambda} ; b\right) \downarrow\right) \cap h\left(k_{(f g)^{n}} \downarrow\right)=h\left(\left(k_{\Lambda} ; b\right) \downarrow \cdot k_{(f g)^{n}} \downarrow\right) \\
& \Rightarrow(x, y) \in h(\emptyset) .
\end{aligned}
$$

The third to last line uses the fact that $k_{\Lambda}$ is the greatest lower bound of $k_{(f g)^{n}} ;(\bar{g} \bar{f})^{n}$ and $a$ in $\mathcal{A}_{n}$, and the last line uses the fact that $k_{\Lambda} ; b$ and $k_{(f g)^{n}}$ have no lower bound in $\mathcal{A}_{n}$. This is a contradiction, so $\mathcal{A}_{n}^{\prime}$ is not representable.

Note that one can write a quasi-equation that is valid in representable SOMs but fails in $\mathcal{A}_{n}^{\prime}$. The antecedent would describe that part of the algebra $\mathcal{A}_{n}^{\prime}$ that we used in the above argument $\left(k_{(f g)^{n}} \downarrow \leq(a ; b) \downarrow \wedge b \downarrow \leq(f g)^{n} \downarrow \wedge \cdots\right)$ and the consequent would be $k_{(f g)^{n}} \downarrow \leq\left(k_{\Lambda} ; b\right) \downarrow$.

Remark 4.12. We can now explain, in outline, why we needed to include the characters $k_{s}$ in our alphabet. A simplified version, say $\mathcal{B}_{n}$, of $\mathcal{A}_{n}$ could be defined without these characters but with a character $c \prec a ; b$ replacing $k_{(f g)^{n}}$, and with appropriate alterations to Definition 4.3 above. That is, we delete all reductions involving characters $k_{s}$ (items (III), (IV) and (V) in Definition 4.3) and include reductions $c \prec a b, a b \prec c \bar{g} g, a b \prec c f \bar{f}$ instead. The problem here is that it would be "too easy" to prove that the algebra $\mathcal{B}_{n}^{\prime}$ was not representable. Very roughly, if a representation did exist then it would contain points $(x, y)$ in the representation of $c \leq a ; b \leq a ;(f g)^{n-1} ; f ; g$, so there would be points $z, z_{1}, z_{2}$ with $(x, z),\left(z, z_{1}\right),\left(z_{1}, z_{2}\right)$ and $\left(z_{2}, y\right)$, respectively, in the representations of $a,(f g)^{n-1}, f$ 
and $g$. Using $\bar{f} ; f, g ; \bar{g} \leq 1^{\prime}$ we could show that $\left(y, z_{2}\right),\left(z_{2}, z_{1}\right)$ belonged, respectively, to the representations of $\bar{g}$ and $\bar{f}$, and so $\left(y, z_{1}\right)$ would belong to the representation of $\bar{g} ; \bar{f}$. But then $\left(x, z_{1}\right)$ would belong to the representations of $a(f g)^{n-1}$ and $c \bar{g} \bar{f}$. Yet these two elements have no lower bound in this modified version of the algebra so we could derive a contradiction. The problem is that we could prove the non-representability of the algebra by considering only five nodes in a claimed representation. With this construction we would be unable to prove that an ultraproduct of our algebras was representable. We add the character $k_{(f g)^{n-1}}$ precisely to act as a lower bound of $a(f g)^{n-1}$ and $c \bar{g} \bar{f}$ and we replace $c$ by $k_{(f g)^{n}}$.

Once we have $k_{(f g)^{n-1}} \leq a(f g)^{n-1}, k_{(f g)^{n}} \bar{g} \bar{f}$ in our alphabet, a similar argument persuades us to include $k_{(f g)^{n-2}} \leq a(f g)^{n-2}, k_{(f g)^{n-1}} \bar{g} \bar{f}$ in our alphabet too and, in the same way, characters $k_{(f g)^{i}}: i \leq n$. Characters of the form $k_{(f g)^{i} f}: i<n$ are not strictly necessary (note that $k_{(f g)^{i} f} \equiv k_{(f g)^{i}} f$ ), but are also included for consistency of notation.

Problem 4.13. It follows from Lemma 2.9 and Theorem 4.11 that the ordered monoid $\mathcal{A}_{n}$ has no principal representation. Does it have an ordered monoid representation?

\section{Towards representability}

Our next aim is to show that a non-principal ultraproduct of the $\mathcal{A}_{n}(n \in \mathbb{N})$ has a principal representation. In the next section, we will describe a winning strategy for $\exists$ on $G_{k}\left(\mathcal{A}_{n}\right)$ for $k<2^{n}$. It will follow that $\exists$ has a winning strategy in the infinite game on the ultraproduct. To describe the winning strategy, we need more on normal forms.

5.1. More on normal forms. Next we state further properties of the system $\left(S_{n}, \leq\right)$. These results are rather technical, and we postpone the proofs to an appendix.

Lemma 5.1. Let $x, y \in(F \cup\{b\})^{*}$ and $x \geq y$. Then either $\operatorname{nf}(x) \stackrel{\psi \bar{\psi} \succ 1^{\prime}}{\longrightarrow} z \geq \operatorname{nf}(y)$ (for some $z$ and some $\psi \in\{f, \bar{g}\}$ ) or $\operatorname{nf}(y)$ is obtained from $\operatorname{nf}(x)$ by a sequence of reductions $(f g)^{n} \succ b$.

Lemma 5.2. Let $x, y, z \in(F \cup\{b\})^{*}$. Suppose $x=\operatorname{nf}(x)$ and $y z=\operatorname{nf}(y z)$ and that $y, z$ are minimal subject to $y z \geq x$ (i.e., if $y^{\prime} \leq y, z^{\prime} \leq z$ and $y^{\prime} z^{\prime} \geq x$ then $y^{\prime} \equiv y$ and $\left.z^{\prime} \equiv z\right)$. Then either

- $y=y_{0} s, z=t z_{0}$, for some $y_{0}, z_{0}, s, t$ where $s t=(f g)^{\text {in }}$ for some $i \in \mathbb{N}$, and $x=y_{0} b^{i} z_{0}$, or

- $y=y_{0} \phi, z=\bar{\phi} z_{0}$ and $y_{0} z_{0} \geq x$, for some $\phi \in F$ and some $y_{0}, z_{0}$. 
Lemma 5.3. Let $e \geq 1^{\prime}$ and $\rho \in \mathcal{A}_{n}$.

(1) Either $k_{(f g)^{n}} e \geq k_{\Lambda} b$ or $e \equiv 1^{\prime}$ (and not both).

(2) Either $k_{\Lambda} b e \geq k_{(f g)^{n}}$ or $e \equiv 1^{\prime}$.

(3) If $\gamma \geq \rho k_{(f g)^{n}}$ but $\gamma \geq \rho k_{\Lambda} b$, then $\gamma \equiv \rho^{+} k_{(f g)^{n}}$ for some $\rho^{+} \geq \rho$.

(4) If $\gamma \geq \rho k_{\Lambda} b$ but $\gamma \geq \rho k_{(f g)^{n}}$, then $\gamma \equiv \rho^{+} k_{\Lambda} b$ for some $\rho^{+} \geq \rho$.

The next step is to define a right and a left quotient $x / \phi$ and $\phi \backslash x$, for $x \in \mathcal{A}_{n}$ and $\phi \in F$. We concentrate on the right quotient as the left quotient is easier. We intend to define $x / \phi$ as a minimal solution $z$ of $z ; \phi \geq x$, i.e., in such a way that $\forall y(y \geq x / \phi \leftrightarrow y ; \phi \geq x)$. Unfortunately this is not possible: there are two inequivalent minimal solutions, $z=k_{(f g)^{n}}$ and $z=k_{\Lambda} b$, to the inequality $z ; \bar{g} \geq k_{(f g)^{n}} \bar{g}$. So we have to define $x / \phi$ as a subset of (rather than a single element of) $\mathcal{A}_{n}$. In one type of case, essentially variants of $\left(k_{(f g)^{n}} \bar{g}\right) / \bar{g}=\left\{k_{(f g)^{n}}, k_{\Lambda} b\right\}, x / \phi$ is a set with two elements, and in all other cases $x / \phi$ is a singleton set. We may identify this singleton with the element of $\mathcal{A}_{n}$ it contains.

Definition 5.4. Let $x \in \mathcal{A}_{n}$. Define $\left.x\right\rfloor$ and $\lfloor x$ from $x$ as follows.

- If $x=x_{0}(f g)^{n}$, for some $x_{0} \in A_{n}$, then $\left.x\right\rfloor=x_{0} b$, else $\left.x\right\rfloor=x$.

- If $x=(f g)^{n} x_{0}$, for some $x_{0} \in A_{n}$, then $\left\lfloor x=b x_{0}\right.$, else $\lfloor x=x$.

Note that both $x\rfloor$ and $\lfloor x$ are in normal form if $x$ is in normal form, hence $x\rfloor,\left\lfloor x \in A_{n}\right.$.

Definition 5.5. Let $x \in \mathcal{A}_{n}$ and let $\phi \in F$. We define $x / \phi$ as follows.

(I) If $\phi \in\{\bar{f}, g\}$, then $x / \phi=\{\operatorname{nf}(x \bar{\phi})\}$ (which we write as $x / \phi=\operatorname{nf}(x \bar{\phi})$ ).

(II) Let $\phi=f$.

(a) If $x=x_{0} f$ (for some $x_{0} \in A_{n}$ ), then $x / f=x_{0}$.

(b) Else $\left(x \neq x_{0} f\right.$ for any $\left.x_{0} \in A_{n}\right) x / f=\operatorname{nf}(x \bar{f})$.

(III) Let $\phi=\bar{g}$.

(a) If $x=x_{0} k_{\Lambda}(f g)^{n-1} f$, for some $x_{0} \in A_{n}$, then $x / \bar{g}=\left\{x_{0} k_{\Lambda} b, x_{0} k_{(f g)^{n}}\right\}$.

(b) If $x=x_{0} \bar{g}$, some $x_{0} \in A_{n}$, then $x / \bar{g}=x_{0}$.

(c) If $x=x_{0} k_{s} f$, some $x_{0} \in A_{n}$ and some initial segment $s f$ of $(f g)^{n}$, then $x / \bar{g}=x_{0} k_{s f g}$.

(d) Else $\left(x \neq x_{0} k_{\Lambda}(f g)^{n-1} f, x_{0} \bar{g}, x_{0} k_{s} f\right.$ for any $\left.\left.x_{0}, s\right) x / \bar{g}=(x g)\right\rfloor$.

Note that $x / \phi$ consists of strings in normal form, hence elements of $A_{n}$. Next we state without proof two rather easy lemmas.

Lemma 5.6. Let $x, z \in \mathcal{A}_{n}$ and $\phi \in F$. If $z \in x / \phi$ then $z ; \phi \geq x$ and $z \leq x ; \bar{\phi}$.

Lemma 5.7. Let $x \in \mathcal{A}_{n}$ and let $\phi \in F$. Then there is $z \in(x ; \phi) / \phi$ with $z \leq x$.

The following theorem summarizes the main features of the right quotient; the proof is in the appendix. 
Theorem 5.8. Let $x, y \in \mathcal{A}_{n}$ and $\phi \in F$.

(1) $x \leq y \Rightarrow(\forall z \in y / \phi)\left(\exists z^{\prime} \in x / \phi\right) z^{\prime} \leq z$.

(2) $y ; \phi \geq x \Leftrightarrow(\exists z \in x / \phi) z \leq y$.

(3) $(\forall z \in y / \phi)\left(\exists z^{\prime} \in(x ; y) / \phi\right) z^{\prime} \leq x ; z$.

Next we define the left quotient.

Definition 5.9. Let $x \in \mathcal{A}_{n}$ and $\phi \in F$. The left quotient $\phi \backslash x$ is defined similarly, but this time we find that $\phi \backslash x$ is always a singleton, so we let $\phi \backslash x \in \mathcal{A}_{n}$ (rather than a subset of $A_{n}$ ).

(I) If $\phi \in\{f, \bar{g}\}$, then $\phi \backslash x=\operatorname{nf}(\bar{\phi} x)$.

(II) If $x=g x_{0}$ (for some $\left.x_{0} \in A_{n}\right)$, then $g \backslash x=x_{0}$, else $\left(x \neq g x_{0}\right.$, any $\left.x_{0}\right)$ $g \backslash x=\bar{g} x$.

(III) If $x=\bar{f} x_{0}$ (for some $x_{0} \in A_{n}$ ), then $\bar{f} \backslash x=x_{0}$, else $\left(x \neq \bar{f} x_{0}\right.$, any $\left.x_{0}\right)$ $\bar{f} \backslash x=\lfloor(f x)$.

Note that the last two cases here are simpler than the corresponding cases in the definition of right quotient. This is because there are no reductions of the form $\alpha k_{s} \succ \beta$, for any $\alpha, s, \beta$. Hence the following theorem has a simpler form than Theorem 5.8.

Theorem 5.10. Let $x, y \in \mathcal{A}_{n}$ and $\phi \in F$.

(1) $x \leq y \Rightarrow \phi \backslash x \leq \phi \backslash y$.

(2) $\phi ; y \geq x \Leftrightarrow y \geq \phi \backslash x$.

(3) $\phi \backslash(x ; y) \leq(\phi \backslash x) ; y$.

We state the following easy lemma without proof:

\section{Lemma 5.11.}

$$
\bar{\phi} ; x \geq \phi \backslash x .
$$

5.2. A strategy. Fix $n>0$ and fix $m>2^{n}$. We aim to show that $\exists$ has a winning strategy in the game $G_{n}\left(\mathcal{A}_{m}\right)$ (see Theorem 5.22 below).

First we define short elements - the intuition is that an edge $(x, y)$ labelled by a short element, a "short" edge, will always have a converse edge $(y, x)$. Thus, $\exists$ has to choose the label for these edges very carefully, e.g., $N(x, y) ; N(y, x) \geq 1^{\prime}$. $\exists$ will not label the converse edge for "long" edges, and this will make the move easier. The "length" of an element is defined relative to the number of remaining rounds of the play.

Definition 5.12. Let $k>0$. 
- An element $x \in \mathcal{A}_{m}$ is called $k$-short if there are $X_{i}, Y_{i} \in \mathcal{A}_{m}: i<k$ such that

$$
x \leq X_{0} ; Y_{0} ; X_{1} ; Y_{1} ; \cdots ; X_{k-1} ; Y_{k-1}
$$

and $\overline{X_{i}} ; X_{i}=Y_{i} ; \overline{Y_{i}}=1^{\prime}$, for each $i<k$. Observe, for $X \neq 1^{\prime}$, that $\bar{X} ; X=$ $1^{\prime} \Longleftrightarrow X \in\{f, \bar{g}\}^{*}$ and $Y ; \bar{Y}=1^{\prime} \Longleftrightarrow Y \in\{\bar{f}, g\}^{*}$.

- We say that $x$ is $k^{-}$-short if $x ; Y$ is $k$-short for some $Y$ with $Y ; \bar{Y}=1^{\prime}$.

- $x$ is called ${ }^{-} k$-short if $X ; x$ is $k$-short for some $X$ with $\bar{X} ; X=1^{\prime}$.

For example, in $\mathcal{A}_{m}$, the element $b$ is $m$-short because $b \leq(f g)^{m}$ (take each $X_{i}=f$ and $\left.Y_{i}=g\right)$ but $b$ is not $(m-1)$-short, nor is it $m^{-}$-short or ${ }^{-} m$-short. Indeed,

$$
s \text { is } k \text {-short (for some } k<m \text { ) } \Rightarrow s \in F^{*} .
$$

Note that if an element is $k$-short, $k^{-}$-short, or ${ }^{-} k$-short, then it is $l$ short for every $l \geq k$. Any member of $F \cup\{\Lambda\}$ is 1 -short, $\bar{f}$ and $g$ are ${ }^{-1} 1$-short, and $\bar{g}$ and $f$ are $1^{-}$-short.

Lemma 5.13. For any $k \in \mathbb{N}$ and any $x \in \mathcal{A}_{m}$ we have that $x$ is $k$-short iff $\left.x\right\rfloor$ is $k$-short iff $\lfloor x$ is $k$-short.

Proof. Recall from Definition 5.4 that $x\rfloor$ is either identical to $x$ or it is defined from $x$ by replacing a final segment $(f g)^{m}$ by $b$. Now, for any $s \in F^{*}$ we have $b \leq s \Longleftrightarrow(f g)^{m} \leq s$, so the lemma follows from this.

We omit the proof of the following easy lemma.

Lemma 5.14. Let $\phi \in F$ satisfy $\bar{\phi} ; \phi=1^{\prime}($ so $\phi \in\{f, \bar{g}\})$ and $x, s \in \mathcal{A}_{m}$. Let $0<k<m$.

(1) $x$ is $k$-short iff $x ; \bar{\phi}$ is $k$-short iff $\phi ; x$ is $k$-short.

(2) $x$ is $-k$-short iff $x ; \bar{\phi}$ is $-k$-short.

(3) $x$ is $k^{-}$-short iff $x ; \phi$ is $k^{-}$-short.

(4) $x$ is $-k$-short iff $\bar{\phi} ; x$ is $-k$-short.

(5) If $s ; x$ is $k$-short (respectively ${ }^{-} k$-short), then $x$ is $k$-short (- $k$-short).

(6) If $x ; s$ is $k$-short (respectively $k^{-}$-short), then $x$ is $k$-short ( $k^{-}$-short).

The strategy is designed to ensure that if there are $r$ rounds left in the game, then the current network is $2^{r}$-good, as defined next.

Definition 5.15. Let $N$ be a partial map $(D \times D) \rightarrow \mathcal{A}_{m}$ (for some set of nodes $D)$ and let $0<k<m$. Say $N$ is $k$-good (respectively $k^{-}$-good, ${ }^{-} k$-good) if, for all $x, y, z \in N$ and all $\alpha, \beta \in \mathcal{A}_{m}$,
(A) $N$ is a consistent network,
(B) if $N(x, y)$ is $k$-short $\left(k^{-}\right.$-short, ${ }^{-} k$-short $)$ then $N(y, x)$ is defined,
(C) if 
(a) $N(x, y), N(y, x)$ are both defined and $\alpha, \beta$ are minimal solutions of $N(x, y) \leq \alpha ; \beta$ (i.e. $\alpha_{0} \leq \alpha \wedge \beta_{0} \leq \beta \wedge \alpha_{0} ; \beta_{0} \geq N(x, y) \rightarrow(\alpha \equiv$ $\left.\left.\alpha_{0} \wedge \beta \equiv \beta_{0}\right)\right)$,

(b) it is not the case that $\alpha=\alpha^{\prime} \phi, \beta=\bar{\phi} \beta^{\prime}$ and $\alpha^{\prime} ; \beta^{\prime} \geq N(x, y)$ (for any $\alpha^{\prime}, \beta^{\prime} \in \mathcal{A}_{m}$ and $\left.\phi \in F\right)$,

(c) it is not the case that $\alpha=\alpha^{\prime} b, \beta=\bar{g} \beta^{\prime}$ nor is it the case that $\alpha=\alpha^{\prime} \bar{f}$, $\beta=b \beta^{\prime}$ (for any $\alpha^{\prime}, \beta^{\prime} \in \mathcal{A}_{m}$ ),

then there is $w \in N$ such that $N(x, w)=\alpha$ and $N(w, y)=\beta$.

Clearly, if $N$ is $k$-good then $N$ is $l$-good for all $l \leq k$.

Note that if $\alpha, \beta \in \mathcal{A}_{m}$ are as in condition $\mathrm{C}$ above, then $\alpha ; \beta=\operatorname{nf}(\alpha \beta)=$ $\alpha \beta$. The next few lemmas include the technical results needed to show that these conditions can be preserved from one round of the game to the next. We will define a number of networks $\left(N^{0}, N^{1}, N^{2}, N^{3}, N^{4}\right)$ and throughout we will adopt the following convention.

Convention: Let $N$ be a network, $x, y \in N, \alpha \in \mathcal{A}_{m}$ and $\phi \in F$. When we write $\beta=\alpha ; N(x, y)$, if $N(x, y)$ is undefined then $\beta$ is also undefined. Similarly, if we write $\beta=\operatorname{nf}(\alpha N(x, y)), \beta=N(x, y) ; \alpha, \beta=N(x, y) / \phi, \beta=\phi \backslash N(x, y)$, etc., if $N(x, y)$ is undefined then $\beta$ is undefined.

Lemma 5.16. Let $1 \leq k<m$. Suppose $N$ is $k$-good, $x, y \in N, \alpha ; \beta \geq N(x, y)$, $N(y, x)$ is not defined, and neither $\alpha$ nor $\beta$ is $k$-short. Then there is a $k$-good extension $N^{0} \supseteq N$ such that there is $z$ with $N^{0}(x, z)=\alpha$ and $N^{0}(z, y)=\beta$.

Indeed, if the witness $z$ is missing from $N$, we define $N^{0}=N^{0}(N, x, y, \alpha, \beta)$ as follows. Let $N^{0}$ have one node, $z$, in addition to those of $N$. To define the labelling of edges incident with $z$, let $w \in N$ be arbitrary.

$$
\begin{aligned}
& N^{0}(z, z)=1^{\prime}, \\
& N^{0}(w, z)=N(w, x) ; \alpha, \\
& N^{0}(z, w)=\beta ; N(y, w) .
\end{aligned}
$$

Proof. Consistency of $N^{0}$ (condition A) is easily checked, using the consistency of $N$ (for condition Id, note that if $\gamma ; \delta \leq 1^{\prime}$, then $\gamma$ and $\delta$ are 1-short). Condition B is trivial since $N^{0}$ has no $k$-short edges incident with the new node $z$ (use Lemma 5.14). Condition C holds because $N^{0}(w, z)$ and $N^{0}(z, w)$ cannot both be defined, else by our convention $N(w, x)$ and $N(y, w)$ would be defined, but we are assuming that $N(y, x)$ is not defined.

Next we deal with the cases where either $\alpha$ or $\beta$ is $k$-short. To handle the case where $\alpha$ is $k$-short we consider, in the next lemma, $\forall$-moves of the form $(N, x, y, \phi, \beta)$ for $\phi \in F$, i.e., we suppose $\alpha=\phi$. The response turns out to be independent of $\beta$. 
We then have to re-use this lemma $|\alpha|$ times to handle more general moves where $\alpha$ is $k$-short.

Lemma 5.18 is to help us with $\forall$-moves where $\beta$ is $k$-short. Note, however, that the lemmas are not exactly symmetrical: the response to $N(x, y, \alpha, \phi)$ does depend on $\alpha$. To compensate we use the assumption that $N(y, x)$ is not defined in Lemma 5.18, whereas this assumption is not needed in Lemma 5.17. To deal with the case where $\beta$ is $k$-short and $N(y, x)$ is defined we need an extra lemma (see $N^{3}$ in Lemma 5.19).

Lemma 5.17. Let $1 \leq k<m$ and let $N$ be a network. Let $x, y \in N$ be such that $N(x, y)$ is defined, and assume $\phi \in F$. Then there is $N^{1} \supseteq N$ and $z$ such that $N(x, z)=\phi$. Furthermore,

(1) if $N$ is ${ }^{-} k$-good and $\phi \in\{\bar{f}, g\}$, then $N^{1}$ is $-k$-good;

(2) if $N$ is $k$-good and $\phi \in\{f, \bar{g}\}$ then $N^{1}$ is $k$-good.

Indeed, if the required witness is missing from $N$, we can define $N^{1}=N^{1}(N, x, \phi)$ with one extra node, $z$ say, and labelling of edges incident with $z$ given by

$$
\begin{aligned}
& N^{1}(z, z)=1^{\prime} \\
& N^{1}(v, z)=N(v, x) ; \phi \\
& N^{1}(z, v)= \begin{cases}\phi \backslash N(x, v) & \text { if } N(y, v) \text { is defined, } \\
\phi ; N(x, v) & \text { otherwise. }\end{cases}
\end{aligned}
$$

Note, in the definition of $N^{1}(z, v)$, that if $N(y, v)$ is defined, then $N(x, v)$ must also be defined, since we are assuming that $N(x, y)$ is defined. Since

$$
\bar{\phi} ; N(x, v) \geq \phi \backslash N(x, v)
$$

(cf. Lemma 5.11), whichever alternative is used in the definition of $N^{1}(z, v)$ we have

$$
N^{1}(z, v) \geq \phi \backslash N(x, v) .
$$

So, by Theorem 5.10 ,

$$
\phi ; N^{1}(z, v) \geq N(x, v) .
$$

Proof. We prove 1 (2 is easier). Suppose $N$ is ${ }^{-} k$-good and the required witness is misssing from $N$. We must show that $N^{1}$ is also ${ }^{-} k$-good. First we check that $N^{1}$ is a consistent network (condition A). Conditions Refl and Trans are obvious. For Comp, let $u, v \in N$ be arbitrary.

$$
\begin{aligned}
N^{1}(u, v) ; N^{1}(v, z) & =N(u, v) ; N(v, x) ; \phi \\
& \geq N(u, x) ; \phi \\
& =N^{1}(u, z) .
\end{aligned}
$$


Also, by Theorem 5.10,

$$
\begin{aligned}
N^{1}(u, z) ; N^{1}(z, v) & \geq N(u, x) ; \phi ;(\phi \backslash N(x, v)) \\
& \geq N(u, x) ; N(x, v) \\
& \geq N^{1}(u, v) .
\end{aligned}
$$

We must also check that $N^{1}(z, u) ; N^{1}(u, v) \geq N^{1}(z, v)$ (whenever $N^{1}(z, u), N^{1}(u, v)$ are both defined). If $N(y, u)$ is undefined then

$$
\begin{aligned}
N^{1}(z, u) ; N^{1}(u, v) & =\bar{\phi} ; N(x, u) ; N(u, v) \\
& \geq \bar{\phi} ; N(x, v) \\
& =N^{1}(z, v) .
\end{aligned}
$$

If $N(z, u), N(u, v)$ are defined and $N(y, u)$ is also defined, then by transitivity of the labelling of $N, N(y, v)$ is also defined, so by Theorem 5.10,

$$
\begin{aligned}
N^{1}(z, u) ; N^{1}(u, v) & =(\phi \backslash N(x, u)) ; N(u, v) \\
& \geq \phi \backslash(N(x, u) ; N(u, v)) \\
& \geq \phi \backslash N(x, v) \\
& =N^{1}(z, v) .
\end{aligned}
$$

For Id, we have $N^{1}(v, z) \neq 1^{\prime}$, since $\phi \in\{\bar{f}, g\}$. Also $N^{1}(z, v)=1^{\prime}$ would imply $N(x, v)=\phi$ (cf. Definition 5.9), but we assumed that $N$ contained no node $w$ satisfying $N(x, w)=\phi$.

We check condition B. If $N^{1}(u, z)=N(u, x) ; \phi$ is ${ }^{-} k$-short, then since $\phi \in\{\bar{f}, g\}$ and by Lemma 5.14(2), $N(u, x)$ is ${ }^{-} k$-short. Since $N$ is ${ }^{-} k$-good, $N(x, u)$ is defined and hence $N^{1}(z, u)$ is also defined. This shows that if $N^{1}(u, z)$ is ${ }^{-} k$-short then $N^{1}(z, u)$ is defined. Now suppose $N^{1}(z, u)$ is ${ }^{-} k$-short. We must show that $N^{1}(u, z)$ is defined. By assumption we have $\phi \in\{\bar{f}, g\}$. So if $N^{1}(z, u)$ is ${ }^{-} k$-short then $N(x, u) \leq \phi ; N^{1}(z, u)$ is also ${ }^{-} k$-short, by Lemma 5.14 . Inductively, $N(u, x)$ must be defined and hence $N^{1}(u, z)$ is also defined. This proves condition B.

For condition C, suppose $N^{1}(u, z), N^{1}(z, u)$ are both defined (hence $N(u, x)$ and $N(x, u)$ are both defined), $N^{1}(u, z)=N(u, x) ; \phi \leq \alpha ; \beta$ and $\alpha, \beta$ satisfy all the hypotheses of condition $\mathrm{C}$. We claim that

$$
\alpha ; \beta=\alpha \beta
$$

(i.e., relative product coincides with concatenation). To prove this, suppose not. There must be a non-empty sequence of replacements (see Definition 4.5) taking us from $\alpha \beta$ to $\operatorname{nf}(\alpha \beta)$. Note that $\alpha, \beta \in(F \cup\{b\})^{*}$ (since $\alpha ; \beta ; N(z, u) \geq 1^{\prime}$ ), so these replacements must be $1,2,3$ or 4 from Definition 4.5 . The first replacement cannot be confined to $\alpha$ (or to $\beta$ ) since $\operatorname{nf}(\alpha)=\alpha$ (and $\operatorname{nf}(\beta)=\beta$ ). Thus the first replacement must replace the concatenation of a non-empty final segment of $\alpha$ and 
a non-empty initial segment of $\beta$. But each of the three possible cases is ruled out by the stipulations for $\alpha, \beta$ in condition $\mathrm{C}: b \bar{g} \rightarrow(f g)^{n-1} f, \bar{f} b \rightarrow g(f g)^{n-1}$ and $\psi \bar{\psi} \rightarrow 1^{\prime}$. This proves the claim.

Hence we can apply Lemma 5.2. Since it is not the case that $\alpha=\alpha^{\prime} \psi, \beta=\bar{\psi} \beta^{\prime}$ and $\alpha^{\prime} \beta^{\prime} \geq N^{1}(u, z)$, that lemma tells us that there are $\alpha_{0}, \beta_{0}, s, t$ and $i \in \mathbb{N}$ with $s t=(f g)^{i m}, \alpha=\alpha_{0} s, \beta=t \beta_{0}$ and $N^{1}(u, z)=\operatorname{nf}(N(u, x) \phi)=\alpha_{0} b^{i} \beta_{0}$.

If $\beta=1^{\prime}$ then the required witness is $z$. Now assume $\beta \neq 1^{\prime}$. There are four cases according to the reductions used in computing $N^{1}(u, z)=\operatorname{nf}(N(u, x) \phi)$ from $N(u, x) \phi$.

- If $N^{1}(u, z)=N(u, x) ; \phi=N(u, x) \phi$, then by the above, $N(u, x) \phi=\alpha_{0} b^{i} \beta_{0}$. Since $\phi \neq b, \beta_{0} \neq 1^{\prime}$ and $\beta_{0}=\beta_{1} \phi$ (for some $\beta_{1}$ ) and $N(u, x)=\alpha_{0} b^{i} \beta_{1} \leq$ $\alpha_{0} s ; t \beta_{1}$, where $s t=(f g)^{i m}$. We have $\alpha_{0} s=\operatorname{nf}\left(\alpha_{0} s\right), t \beta_{1}=\operatorname{nf}\left(t \beta_{1}\right)$, and, since $s t=(f g)^{i m}$, it is not the case that $\alpha_{0} s=\alpha_{2} \psi, t \beta_{1}=\bar{\psi} \beta_{2}$ (any $\alpha_{2}, \beta_{2}$ ), so by condition $\mathrm{C}$ for $N$, there is $w \in N$ with $N(u, w) \leq \alpha_{0} s, N(w, x) \leq t \beta_{1}$ and so $N^{1}(w, z) \leq t \beta_{1} ; \phi=t \beta_{0}=\beta$, and $w$ is the witness we need in $N^{1}$.

- If $N(u, x)=\rho \bar{\phi}$ and $\bar{\phi} ; \phi=1^{\prime}$ then $N^{1}(u, z)=\operatorname{nf}(\rho \bar{\phi} \phi)=\rho$. Then $\alpha ; \beta \geq \rho$ implies $\alpha ; \beta \bar{\phi} \geq \rho \bar{\phi}=N(u, x)$. It is not difficult to show that the requirements for condition $\mathrm{C}$ are still met by $\alpha$ and $\operatorname{nf}(\beta \bar{\phi})=\beta \bar{\phi}$, so inductively there is $w \in N$ with $N(u, w) \leq \alpha, N(w, x) \leq \beta \bar{\phi}$. Hence $N^{1}(w, z) \leq \beta ; \bar{\phi} ; \phi=\beta$ and $w$ is the required witness in $N^{1}$.

- If $N(u, x)=\rho b$ (for some $\rho$ ) and $\phi=\bar{g}$ then $N^{1}(u, z)=\operatorname{nf}(\rho b \bar{g})=\rho(f g)^{n-1} f$. So $\alpha ; \beta ; g \geq N^{1}(u, z) ; g=\rho(f g)^{n} \geq N(u, x)$. It is easy to check that the requirements for condition $\mathrm{C}$ are met by $\alpha, \beta g$, so inductively there is $w \in N$ with $N(u, w) \leq \alpha$ and $N(w, x) \leq \beta g$. Hence $N^{1}(w, z) \leq \beta ; g ; \bar{g}=\beta$ and $w$ is the required witness.

- The case where $N(u, x)=\rho b$ and $\phi=f$ is similar.

Also, consider the case where $N^{1}(u, z), N^{1}(z, u)$ are defined and $N^{1}(z, u) \leq \alpha ; \beta$. If $N^{1}(z, u)=\bar{\phi} ; N(x, u)$ then this is entirely similar to the previous case. So suppose $N(y, u)$ is defined and $N^{1}(z, u)=\phi \backslash N(x, u) \leq \alpha ; \beta$ where $\alpha \beta=\operatorname{nf}(\alpha \beta)$, and it is not the case that $\alpha=\alpha^{\prime} \psi, \beta=\bar{\psi} \beta^{\prime}$ and $\alpha^{\prime} \beta^{\prime} \geq N^{1}(z, u)$ (any $\alpha^{\prime}, \beta^{\prime}$ and and $\psi \in F)$. Since $N^{1}(z, u)=\phi \backslash N(x, u) \leq \alpha \beta$ we have $N(x, u) \leq \operatorname{nf}(\phi \alpha) ; \beta=\operatorname{nf}(\phi \alpha \beta)$ so, inductively, there is $w \in N$ such that $N(x, w) \leq \operatorname{nf}(\phi \alpha)$, and $N(w, u) \leq \beta$. Since $N(y, w) \leq N(y, u) ; N(u, x) ; N(x, w)$ is defined, we have $N^{1}(z, w)=\phi \backslash N(x, w) \leq$ $\phi \backslash(\phi ; \alpha) \leq \alpha$ (by Theorem 5.10), so $w$ is the required witness in $N^{1}$. This proves condition $\mathrm{C}$.

Part 2 is proved similarly.

Lemma 5.18. Let $N$ be a network, $x, y \in N$ and $\alpha ; \phi \geq N(x, y)$, for some $\alpha \in \mathcal{A}_{m}$ and $\phi \in F$. Suppose that $N(y, x)$ is not defined. Then there is an extension $N^{2} \supseteq N$ such that there is $z$ with $N^{2}(x, z) \leq \alpha$ and $N^{2}(z, y)=\phi$. Further, 
(1) if $N$ is $k$-good and $\phi \in\{\bar{f}, g\}$ then $N^{2}$ is k-good;

(2) if $N$ is $k^{-}$-good and $\phi \in\{f, \bar{g}\}$ then $N^{2}$ is $k^{-}$-good.

If the required witness is missing from $N$, we define $N^{2}=N^{2}(N, x, \alpha, \phi)$ with exactly one node, $z$, in addition to those of $N$. For the labelling of edges incident with $z$, let $N^{2}(z, z)=1^{\prime}$. Let $w \in N$ be arbitrary.

$$
N^{2}(z, w)=\phi ; N(y, w)
$$

Let

$$
N^{2}(w, z) \begin{cases}\in N(w, y) / \phi & \text { if } N(w, x) \text { is defined } \\ =N(w, y) ; \bar{\phi} & \text { otherwise. }\end{cases}
$$

With the first alternative in (2) we must write ' $\in$ ' instead of ' $=$ ' because $N(w, y) / \phi$ is a set, not necessarily a singleton. If it is not a singleton then we have not uniquely defined $N^{2}(w, z)$ (yet). If $N(w, y) / \phi$ has two elements then $N(w, y)=$ $\rho k_{\Lambda}(f g)^{m-1} f$ (see Definition 5.5.III $(a)$ ) and $\phi=\bar{g}$, some $\rho$, and

$$
N(w, y) / \bar{g}=\left(\rho k_{\Lambda}(f g)^{m-1} f\right) / \bar{g}=\left\{\rho k_{\Lambda} b, \rho k_{(f g)^{m}}\right\} .
$$

Note that $N(w, x) ; \alpha ; \bar{g} \geq N(w, x) ; N(x, y) \geq N(w, y)$ so by Theorem 5.8 there is $\gamma \in N(w, y) / \bar{g}$ with $N(w, x) ; \alpha \geq \gamma$. I.e.

$$
N(w, x) ; \alpha \geq \rho k_{(f g)^{m}} \quad \text { or } \quad N(w, x) ; \alpha \geq \rho k_{\Lambda} b .
$$

Let

$$
N^{2}(w, z)= \begin{cases}\rho k_{\Lambda} b & \text { if } N(w, x) ; \alpha \geq \rho k_{\Lambda} b \\ \rho k_{(f g)^{m}} & \text { otherwise. }\end{cases}
$$

With $N^{2}$ thus defined we have $N^{2}(x, z) \leq \alpha$ and $N^{2}(z, y)=\phi$.

Proof. Again assume that the required witness is missing from $N$.

Note that $N^{2}(u, z) ; \phi \geq N^{2}(u, y)$ in all cases. The proof is roughly similar to the proof of Lemma 5.17, but the proof of consistency is rather more complicated because $x / \phi$ is not always a singleton set. Suppose $N$ is $k$-good and $\phi \in\{\bar{f}, g\}$. We must show that $N^{2}$ is also $k$-good.

First we check the consistency of $N^{2}$. Trans and Refl are immediate. Condition Id follows from the following observation. If $1^{\prime} \geq N^{2}(z, w)=\phi ; N(y, w)$, then we have either $N(w, y) \leq \bar{f}$ or $N(w, y) \leq g$. In either case, we get $N(x, w) \leq$ $N(x, y) ; N(y, w) \leq \alpha ; \phi ; \bar{\phi} \leq \alpha$, i.e., the required witness is present in $N$. Similarly, if $N(w, z)=N(w, y) ; \bar{\phi}$, then $N(w, y) \in\{\bar{f}, g\}$, whence $N(x, w) \leq \alpha$ as above. Finally, note that if $N^{2}(z, w) \in N(w, y) / \phi$, then $1^{\prime} \geq N(w, z)$ is impossible.

Next we check Comp. Let $u, v \in N$ and suppose $(u, v)$ is labelled in $N$. Then

$$
\begin{array}{rlrl}
N^{2}(u, z) ; N^{2}(z, v) & =N^{2}(u, z) ;(\phi ; N(y, v)) & & \text { by }(1) \\
& \geq N(u, y) ; N(y, v) & & \text { since } N^{2}(u, z) ; \phi \geq N(u, y) \\
& \geq N^{2}(u, v) . &
\end{array}
$$


Similarly,

$$
\begin{aligned}
N^{2}(z, u) ; N^{2}(u, v) & =\phi ; N(y, u) ; N(u, v) \\
& \geq \phi ; N(y, v) \\
& =N^{2}(z, v) .
\end{aligned}
$$

Finally, we must check that $N^{2}(u, v) ; N^{2}(v, z) \geq N^{2}(u, z)$. If $(v, x)$ is unlabelled in $N$ then $N^{2}(v, z)=N(v, y) ; \bar{\phi}$, by (2). In that case

$$
N^{2}(u, v) ; N^{2}(v, z)=N(u, v) ; N(v, y) ; \bar{\phi} \geq N(u, y) ; \bar{\phi} \geq N^{2}(u, z)
$$

by Lemma 5.6. So assume that $(v, x)$ is labelled in $N$ (hence $(u, x)$ is also labelled, since in the current case $(u, v)$ is labelled). By $(2), N^{2}(u, z) \in N(u, y) / \phi$. If $N(u, y) / \phi$ is a singleton then $N^{2}(u, z)=N(u, y) / \phi$. Since

$$
\begin{aligned}
\left(N(u, v) ; N^{2}(v, z)\right) ; \phi & =N(u, v) ;\left(N^{2}(v, z) ; \phi\right) \\
& \geq N(u, v) ; N(v, y) \\
& \geq N(u, y),
\end{aligned}
$$

it follows by Theorem 5.8 that

$$
N(u, v) ; N^{2}(v, z) \geq N(u, y) / \phi=N^{2}(u, z) .
$$

That leaves the situation where $N(u, y) / \phi$ is not a singleton. In this case $\phi=\bar{g}$ and there is $\rho$ such that $N(u, y)=\rho k_{\Lambda}(f g)^{m-1} f$. We have

$$
N(u, y) / \phi=\left\{\rho k_{(f g)^{m}}, \rho k_{\Lambda} b\right\} .
$$

Now $\left(N(u, v) ; N^{2}(v, z)\right) ; \phi \geq N(u, v) ; N(v, y) \geq N(u, y)$, so by Theorem 5.8 there is $\gamma \in\left\{\rho k_{(f g)^{m}}, \rho k_{\Lambda} b\right\}$ with

$$
N(u, v) ; N^{2}(v, z) \geq \gamma .
$$

By $(2), N^{2}(u, z) \in\left\{\rho k_{\Lambda} b, \rho k_{(f g)^{m}}\right\}$. If $N^{2}(u, z)=\gamma$ we are done. The two remaining possibilities we have to consider (and eliminate) are

(i) $N^{2}(u, z)=\rho k_{(f g)^{m}}, N^{2}(u, v) ; N^{2}(v, z) \geq \rho k_{\Lambda} b$

but $N^{2}(u, v) ; N^{2}(v, z) \nsupseteq \rho k_{(f g)^{m}} ;$

(ii) $N^{2}(u, z)=\rho k_{\Lambda} b, N^{2}(u, v) ; N^{2}(v, z) \geq \rho k_{(f g)^{m}}$

but $N^{2}(u, v) ; N^{2}(v, z) \nsupseteq \rho k_{\Lambda} b$.

For (i), suppose $N^{2}(u, z)=\rho k_{(f g)^{m}}$. By (4), $N(u, x) ; \alpha \nsupseteq \rho k_{\Lambda} b$ and, by (3), $N(u, x) ; \alpha \geq \rho k_{(f g)^{m}}$. By Lemma 5.3, $N(u, x) ; \alpha=\rho^{+} k_{(f g)^{m}}$, for some $\rho^{+} \geq \rho$. Hence, either $\alpha=\alpha_{0} k_{(f g)^{m}}$ (for some $\alpha_{0}$ ), or $N(u, x)=\rho^{+} k_{(f g)^{m}} \bar{\alpha}$ and $\bar{\alpha} ; \alpha=1^{\prime}$. In the former case, $N^{2}(u, v) ; N^{2}(v, z) \leq N(u, v) ; N(v, x) ; \alpha_{0} k_{(f g)^{m}}$. Since $k_{(f g)^{m}}$ is minimal with respect to $<$, this means that $N^{2}(u, v) ; N^{2}(v, z) \geq \rho k_{\Lambda} b$ is impossible. But we are assuming that $N^{2}(u, v) ; N^{2}(v, z) \nsupseteq \rho k_{(f g)^{m}}$. This contradicts (5). For the latter case, $\bar{\alpha} ; \alpha=1^{\prime}$ so $\alpha \in\{f, \bar{g}\}^{*}$ and $\phi=\bar{g}$. So $N(x, y) \leq \alpha ; \bar{g} \in\{f, \bar{g}\}^{*}$ is 
1-short. By condition B for $N, N(y, x)$ is defined, contrary to the assumption in the lemma. Thus case (i) cannot happen.

Now consider possibility (ii) and suppose

$$
N^{2}(u, z)=\rho k_{\Lambda} b \text { but } N^{2}(u, v) ; N^{2}(v, z) \nsupseteq \rho k_{\Lambda} b .
$$

By $(5), N^{2}(u, v) ; N^{2}(v, z) \geq \rho k_{(f g)^{m}}$. By Lemma 5.3, $N^{2}(u, v) ; N^{2}(v, z)=$ $\rho^{+} k_{(f g)^{m}}$, for some $\rho^{+} \geq \rho$. The character $k_{(f g)^{m}}$ belongs either to $N^{2}(u, v)$ or $N^{2}(v, z)$. In the former case, $N^{2}(u, v)=\rho^{+} k_{(f g)^{m}} s$ and $N^{2}(v, z)=\bar{s}$ for some $s$ with $s ; \bar{s}=1^{\prime}$. This means that $N^{2}(v, y) \leq N^{2}(v, z) ; \bar{g}=\bar{s} ; \bar{g}$ is 1-short so $N^{2}(y, v)$ is defined. Hence $N(v, x)$ cannot be defined, else $N(y, x)$ would be defined against our assumption. If $N(v, x)$ is undefined, then $N^{2}(v, z)=N(v, y) ; g$. By Lemma 5.3, this contradicts our assumption that $N^{2}(u, v) ; N^{2}(v, z) \nsupseteq \rho k_{\Lambda} b$.

The other case is where the character $k_{(f g)^{m}}$ belongs to $N^{2}(v, z)$. Say $N(u, v)=$ $\rho_{1}, N^{2}(v, z)=\rho_{2} k_{(f g)^{m}} ; e$ for some $\rho_{1}, \rho_{2}, e$ where $\rho_{1} ; \rho_{2} \geq \rho$ and $e \geq 1^{\prime}$, but $N^{2}(u, v) ; N^{2}(v, z) \nsupseteq \rho k_{\Lambda} b$. By Lemma $5.3, e=1^{\prime}$, so $N^{2}(v, z)=\rho_{2} k_{(f g)^{m}}$. Also, by (4), $N(v, x) ; \alpha \nsupseteq \rho_{2} k_{\Lambda} b$. So, by (3), $N(v, x) ; \alpha \geq \rho_{2} k_{(f g)^{m}}$ and, by Lemma 5.3 , $N(v, x) ; \alpha=\rho_{2}^{+} k_{(f g)^{m}}$, for some $\rho_{2}^{+} \geq \rho_{2}$. The character $k_{(f g)^{m}}$ belongs either to $N(v, x)$ or $\alpha$. In the latter case we must have $N(u, x) ; \alpha \nsupseteq \rho k_{\Lambda} b$, but this contradicts (4) and the assumption that $N^{2}(u, z)=\rho k_{\Lambda} b$. In the former case we have $N(v, x)=\rho_{2}^{+} k_{(f g)^{m}} s$ and $s ; \alpha=1^{\prime}$. Hence $\alpha \in\{f, \bar{g}\}^{*}$ is 1 -short and $N(x, y) \leq \alpha \bar{g}$ is also 1-short. By condition B for $N, N(y, x)$ must be defined, contrary to the assumption in the lemma. Thus case (ii) cannot happen.

This proves that $N^{2}$ is consistent (condition A). The proofs of conditions B and C for $N^{2}$ are similar to the corresponding proofs for Lemma 5.17.

In the next lemma we define two extensions $N^{3}, N^{4}$ to $N$. Each of them has a witness $z$ satisfying $N^{3}(x, z)=N^{4}(x, z)=\phi$, for some specified $x \in N$ and $\phi \in F$.

Lemma 5.19. Let $1<k<m$, let $N$ be a $k$-good network, $\phi \in F$ and $x \in N$. Then there are extensions $N^{3}$ and $N^{4}$ of $N$ such that $N^{3}(x, z)=N^{4}(x, z)=\phi$ for some $z$, and $N^{3}$ is $k^{-}$-good and $N^{4}$ is $k$-good.

If the required witness is missing from $N$, we define $N^{3}=N^{3}(N, x, \phi)$ and $N^{4}=N^{4}(N, x, \phi)$, each with a single node, $z$ say, in addition to those of $N$. Of course, $N^{3}(z, z)=N^{4}(z, z)=1^{\prime}$. To label other edges incident with $z$, let $u \in N$ be arbitrary.

$$
\begin{aligned}
& N^{3}(u, z) \in N(u, x) / \bar{\phi} \\
& N^{3}(z, u)=\bar{\phi} ; N(x, u) \\
& N^{4}(u, z)=N(u, x) ; \phi
\end{aligned}
$$




$$
N^{4}(z, u)=\bar{\phi} ; N(x, u)
$$

In the definition of $N^{3}(u, z)$, if $N(u, x) / \bar{\phi}$ is a set with two elements, say $N^{3}(u, x) / \bar{\phi}=\left\{\rho k_{\Lambda} b, \rho k_{(f g)^{m}}\right\}$, for some $\rho$, then we let $N^{3}(u, z)=\rho k_{\Lambda} b$.

Proof. Consistency of $N^{3}$ and $N^{4}$ is easy to check (use Theorem 5.8 for $N^{3}$ ).

The proofs of conditions B and C for $N^{3}, N^{4}$ are the same as the proofs of the same conditions in Lemma 5.17.

Lemma 5.20. Let $m>k \geq 1$, let $N$ be $k$-good, $x, y \in N$ and suppose $N(x, y)$ and $N(y, x)$ are both defined. If $\alpha ; \beta \geq N(x, y)$ then there is a $(k-1)$-good $N^{\prime} \supseteq N$ containing a node $z$ such that $N^{\prime}(x, z) \leq \alpha$ and $N^{\prime}(z, y) \leq \beta$.

Proof. We can assume that $\alpha, \beta$ are minimal, i.e. if $\alpha^{\prime} \leq \alpha, \beta^{\prime} \leq \beta$ and $\alpha^{\prime} ; \beta^{\prime} \geq$ $N(x, y)$ then $\alpha^{\prime}=\alpha$ and $\beta^{\prime}=\beta$.

If (i) $\alpha=\alpha_{0} b s, \beta=\bar{s} \bar{g} \beta_{0}$ and $\alpha_{0} b ; \bar{g} \beta_{0} \geq N\left(x, y\right.$ ), or (ii) $\alpha=\alpha_{0} \bar{f} s$ and $\beta=\bar{s} b \beta_{0}$ and $\alpha_{0} \bar{f} ; b \beta_{0} \geq N(x, y)$, for some $\alpha_{0}, \beta_{0}$ and some $s \in F^{*}$, then we say that $(\alpha, \beta)$ is dangerous.

For any character $c \in \Sigma_{m}$ define the $\operatorname{rank} \rho(c) \in \mathbb{N}$ by

$$
\rho(c)= \begin{cases}1 & \text { if } c \in F \\ 2 m & \text { if } c=b \\ 0 & \text { otherwise }\end{cases}
$$

For any string $s=s_{0} s_{1} \cdots s_{j-1}$ over $\Sigma_{m}$ let the rank of $s$ be defined by $\rho(s)=$ $\Sigma_{i<j} \rho\left(s_{i}\right)$. We will prove the lemma by strong induction over $\rho(\alpha)+\rho(\beta)$.

Induction hypothesis: Assume $N$ is $k$-good. Let $l \in \mathbb{N}$, and assume that $N(x, y), N(y, x)$ are both defined. If $\alpha_{1} ; \beta_{1} \geq N(x, y)$, and $\rho\left(\alpha_{1}\right)+\rho\left(\beta_{1}\right)<l$, then there is a $(k-1)$-good $N^{\prime} \supseteq N$ containing a node $z$ such that $N^{\prime}(x, z) \leq \alpha_{1}$ and $N^{\prime}(z, y) \leq \beta_{1}$. Furthermore, if $\left(\alpha_{1}, \beta_{1}\right)$ is not dangerous then there is a $k$-good $N^{\prime} \supseteq N$ with a node $z$ such that $N^{\prime}(x, z) \leq \alpha_{1}$ and $N^{\prime}(z, y) \leq \beta_{1}$.

Now we consider the case where $\rho(\alpha)+\rho(\beta)=l$. Suppose that none of the following is true for any $\psi \in F$ and any $\alpha^{\prime}, \beta^{\prime}$ :

- $\alpha=\alpha^{\prime} \psi, \beta=\bar{\psi} \beta^{\prime}$ and $\alpha^{\prime} ; \beta^{\prime} \geq N(x, y)$,

- $\alpha=\alpha^{\prime} b$ and $\beta=\bar{g} \beta^{\prime}$,

- $\alpha=\alpha^{\prime} \bar{f}$ and $\beta=b \beta^{\prime}$.

Since, under these conditions, $(\alpha, \beta)$ is not dangerous we must find a $k$-good $N^{\prime} \supseteq N$ with a suitable witness node. Well, by condition $\mathrm{C}$ of Definition 5.15 , there is $w \in N$ with $N(x, w) \leq \alpha$ and $N(w, y) \leq \beta$ so we may let $N^{\prime}=N$. This is $k$-good, as required.

Now let $\alpha=\alpha^{\prime} \psi, \beta=\bar{\psi} \beta^{\prime}$ and $\alpha^{\prime} ; \beta^{\prime} \geq N(x, y)$, for some $\psi \in F$ and some $\alpha^{\prime}, \beta^{\prime}$. Since $\rho\left(\alpha^{\prime}\right)+\rho\left(\beta^{\prime}\right)=\rho(\alpha)-1+\rho(\beta)-1$, we can use our induction hypothesis. So there is a $(k-1)$-good $N^{+} \supseteq N$ with a node $w$ such that $N^{+}(x, w) \leq \alpha^{\prime}$ and 
$N^{+}(w, y) \leq \beta^{\prime}$. Let $N^{\prime}=N^{4}\left(N^{+}, w, \psi\right)$, defined in Lemma 5.19. By that lemma $N^{\prime}$ is also $(k-1)$-good.

Suppose that $\left(\alpha^{\prime} \psi, \bar{\psi} \beta^{\prime}\right)$ is not dangerous. Then $\left(\alpha^{\prime}, \beta^{\prime}\right)$ is not dangerous either so by the induction hypothesis there is a $k$-good $N^{+} \supseteq N$ with a node $w$ such that $N^{+}(x, w) \leq \alpha^{\prime}$ and $N^{+}(w, y) \leq \beta^{\prime}$. As above we let $N^{\prime}=N^{4}\left(N^{+}, w, \psi\right)$ and by Lemma 5.19 this is $k$-good.

Let $\alpha=\alpha^{\prime} b$ and $\beta=\bar{g} \beta^{\prime}$. Since $\left(\alpha^{\prime} b, \bar{g} \beta^{\prime}\right)$ is dangerous we are only required to find a $(k-1)$-good $N^{+} \supseteq N$ with the required witness. We have $\alpha ; \beta \equiv$ $\alpha^{\prime} ;(f g)^{m-1} f ; \beta^{\prime} \geq N(x, y)$. Since $\rho\left(\alpha^{\prime}(f g)^{m-1} f\right)+\rho\left(\beta^{\prime}\right)<\rho(\alpha)+\rho(\beta)$ we can use our induction hypothesis. If $\left(\alpha^{\prime}(f g)^{m-1} f, \beta^{\prime}\right)$ is not dangerous then there is a $k$-good $N^{+} \supseteq N$ with a node $w$ such that $N^{+}(x, w) \leq \alpha^{\prime}(f g)^{m-1} f$ and $N^{+}(w, y) \leq$ $\beta^{\prime}$. Let $N^{\prime}=N^{3}\left(N^{+}, w, \bar{g}\right)$. By Lemma $5.19, N^{\prime}$ is $(k-1)$-good and it contains a node $z$ such that $N^{\prime}(x, z)=N^{+}(x, w) / \bar{g} \leq\left(\alpha^{\prime}(f g)^{m-1} f\right) / \bar{g} \leq \alpha^{\prime} b=\alpha$ and $N^{\prime}(z, y)=\bar{g} ; N^{+}(w, y) \leq \bar{g} \beta^{\prime}=\beta$.

Now suppose $\left(\alpha^{\prime}(f g)^{m-1} f, \beta^{\prime}\right)$ is dangerous. Then $\beta^{\prime}=\overline{(f g)^{m-1} f} \beta_{0}$ and $\alpha^{\prime} ; \beta_{0} \geq N(x, y)$, for some $\beta_{0}$. By the induction hypothesis there is a $(k-1)$ good $N_{0} \supseteq N$ with a node $w$ such that $N_{0}(x, w) \leq \alpha^{\prime}$ and $N_{0}(w, y) \leq \beta_{0}$. Next we make a sequence of $(k-1)$-good extensions $N_{0} \subseteq N_{1} \subseteq \cdots \subseteq N_{2 m-1}$ as follows. Let $z_{0}=w$, let $i<2 m-1$ and suppose we have defined a $(k-1) \operatorname{good} N_{i}$ containing a node $z_{i}$ such that $N_{i}\left(z_{0}, z_{1}\right)=f, N_{i}\left(z_{1}, z_{2}\right)=g, \ldots, N_{i}\left(z_{i-1}, z_{i}\right)=\phi$, where $\phi$ is the $i$ th character of $(f g)^{m-1} f$. Let $\phi^{\prime}$ be the $(i+1)$ th character of $(f g)^{m-1} f$. Let $N_{i+1}=N^{4}\left(N_{i}, z_{i}, \phi^{\prime}\right)$. By Lemma $5.19 N_{i+1}$ is $(k-1)$-good and it contains a node $z_{i+1}$ such that $N_{i+1}\left(z_{i}, z_{i+1}\right)=\phi^{\prime}$. Thus we can define a $(k-1)$-good $N_{2 m-1} \supseteq N_{0}$ with a node $z_{2 m-1}$ such that $N_{2 m-1}\left(u, z_{2 m-1}\right)=$ $N_{0}\left(u, z_{0}\right) ;(f g)^{m-1} f$ and $N_{2 m-1}\left(z_{2 m-1}, u\right)=\overline{(f g)^{m-1} f} ; N_{0}\left(z_{0}, u\right)$. Finally, let $N^{\prime}=N^{3}\left(N_{2 m-1}, z_{2 m-1}, g\right)$. Lemma 5.19 merely tells us that this is $(k-2)$-good, but this is not good enough. In fact $N^{\prime}$ will be $(k-1)$-good. To see this, let $z \in N^{\prime}$ satisfy $N^{\prime}\left(z_{2 m-1}, z\right)=g$. Suppose for contradiction that $N^{\prime}$ is not $(k-1)$-good. Since $N_{2 m-1}$ is $(k-1)$-good, this means that there is $u \in N_{2 m-1}$ such that either (a) $N^{\prime}(u, z)$ is $(k-1)$-short but $N^{\prime}(z, u)$ is not defined, (b) $N^{\prime}(z, u)$ is $(k-1)$-short but $N^{\prime}(u, z)$ is not defined or (c) $N^{\prime}(u, z), N^{\prime}(z, u)$ are both defined but $N^{\prime}$ contains no witness for $\alpha, \beta$ as in condition $\mathrm{C}$ of Definition 5.15. Observe that if $u \in N_{0}$ then $N^{\prime}(u, z)=\left(N_{0}\left(u, z_{0}\right) ;(f g)^{m-1} f\right) / \bar{g} \leq N_{0}\left(u, z_{0}\right) ;\left((f g)^{m-1} f / \bar{g}\right)=N_{0}\left(u, z_{0}\right) ; b$ by Theorem 5.8. By Lemma 5.14 this is not $(k-1)$-short (since $k<m, b$ is not $k$-short). Also $N^{\prime}(z, u)$ is not $(k-1)$-short if $u \in N_{0}$. So for (a) and (b) we can suppose that $u \in\left\{z_{1}, z_{2}, \ldots, z_{2 m-1}\right\}$. But then, by construction of $N^{\prime}$, we know that $N^{\prime}(z, u)$ is defined (also $N^{\prime}(u, z)$ is defined). For (c), if $N^{\prime}(u, z), N^{\prime}(z, u)$ are both defined and $u \in\left\{z_{1}, \ldots, z_{2 m-1}\right\}$ and $\alpha, \beta$ are as in condition $\mathrm{C}$ of Definition 5.15, then a suitable witness can be found in $\left\{z_{1}, \ldots, z_{2 m-1}\right\}$. On the other hand, if $u \in N_{0}$, then since $N^{\prime}(u, z), N^{\prime}(z, u)$ are both defined, we must have $N_{0}\left(u, z_{0}\right), N_{0}\left(z_{0}, u\right)$ both defined 
and $N^{\prime}(u, z)=N_{0}\left(u, z_{0}\right) ; b, N^{\prime}(z, u)=\overline{(f g)^{m}} ; N_{0}\left(z_{0}, u\right)$. If $\alpha ; \beta \geq N^{\prime}(u, z)$ as in condition $\mathrm{C}$ of Definition 5.15, then either $\alpha=\alpha_{0} ; b$ and $\beta=\overline{(f g)^{m}} ; \beta_{0}$, in which case the required witness can be found by considering $\alpha_{0}, \beta_{0}$ in $N_{0}$, or $\alpha=\alpha_{0} ; s$ and $\beta=t$ for some $s, t$ with $s t=(f g)^{m}$, in which case a suitable witness can be found in $\left\{z_{1}, \ldots, z_{2 m-1}\right\}$. The case $\alpha ; \beta \geq N^{\prime}(z, u)$ is similar. This proves that $N^{\prime}$ is in fact $(k-1)$-good. We have $N^{\prime}(x, z)=N_{2 m-1}\left(x, z_{2 m-1}\right) / \bar{g} \leq\left(\alpha^{\prime}(f g)^{2 m-1} f\right) / \bar{g} \leq$ $\alpha^{\prime} b=\alpha$ and $N^{\prime}(z, y)=\bar{g} ; \overline{(f g)^{m-1} f} \beta_{0} \leq \bar{g} \beta^{\prime}=\beta$, as required.

The case where $\alpha=\alpha^{\prime} \bar{f}$ and $\beta=b \beta^{\prime}$ is similar, but instead of using $N^{3}$ in the construction of $N^{\prime}$ we use $N^{1}$ from Lemma 5.17.

Putting these together we get

Lemma 5.21. Let $2^{r}<m$ and $N$ be $2^{r}$-good. For any $\forall$-move $(N, x, y, \alpha, \beta)$ there is a legal $\exists$-response $N^{\prime} \supseteq N$ and $N^{\prime}$ is $2^{r-1}$-good.

Proof. First suppose $N(y, x)$ is undefined. If neither $\alpha$ nor $\beta$ is $2^{r-1}$-short, then by Lemma 5.16 there is a $2^{r-1}$-good $N^{0} \supseteq N$ making a legal response to the move.

Suppose $\alpha \leq X_{0} ; Y_{0} ; \cdots ; X_{k-1} ; Y_{k-1}$ is $2^{r-1}$ short, where $k \leq 2^{r-1}$ and $X_{i} \in$ $\{f, \bar{g}\}^{*}, Y_{i} \in\{\bar{f}, g\}^{*}$ for $i<k$. Since $N$ is $2^{r}$-good it is certainly ${ }^{-} 2^{r}$-good. Using Lemma $5.17\left|X_{0}\right|$ times, there is a ${ }^{-} 2^{r}$-good $N^{+} \supseteq N$ with a node $z_{0}$ such that $N^{+}\left(x, z_{0}\right) \leq X_{0}, N^{+}\left(z_{0}, y\right) \leq Y_{0} ; X_{1} ; \cdots ; Y_{k-1} ; \beta$. Since $N^{+}$is ${ }^{-} 2^{r}$-good it is certainly $\left(2^{r}-1\right)$-good. Using the same lemma $\left|Y_{0}\right|$ times again, there is a $\left(2^{r}-1\right) \operatorname{good} N^{*} \supseteq N^{+}$with a node $z_{1}$ such that $N^{*}\left(z_{1}, z_{2}\right) \leq Y_{0}$ and $N^{*}\left(z_{2}, y\right) \leq$ $X_{1} ; Y_{1} ; \cdots ; Y_{k-1} ; \beta$. Continuing like this we obtain a $2^{r}-2^{r-1}=2^{r-1}$-good $N^{\prime} \supseteq N$ with a node $z$ such that $N^{\prime}(x, z) \leq X_{0} ; Y_{0} ; \cdots ; Y_{k-1}$ and $N^{\prime}(z, y) \leq \beta$.

Similarly, if $\beta$ is $2^{r-1}$-short, then repeated use of Lemma 5.18 shows that there is a $2^{r-1}$-good $N^{\prime} \supseteq N$ making a legal response to the move.

Now suppose that $N(y, x)$ is defined. By Lemma 5.20 there is a $\left(2^{r}-1\right)$-good legal response $N^{\prime} \supseteq N$ to the move $(N, x, y, \alpha, \beta)$. Since $N^{\prime}$ is $\left(2^{r}-1\right)$-good and $r \geq 1$, it is certainly $2^{r-1}$-good.

Theorem 5.22. $\exists$ has a winning strategy in $G_{n}\left(\mathcal{A}_{m}\right)$ for $2^{n}<m$.

Proof. We define a strategy for $\exists$ in $G_{n}\left(\mathcal{A}_{m}\right)$. We will design the strategy so that if $N_{t}$ is played in round $t$ (so there are $r=n-t$ rounds left in the game) then $N_{t}$ is a $2^{r}$-good network.

In the initial round (round zero) let $\forall$ play $\gamma$. If $\gamma$ is not $2^{n}$-short then let $N_{0}$ have exactly two nodes, $x$ and $y$ and labelling

$$
\begin{aligned}
& N_{0}(x, x)=N_{0}(y, y)=1^{\prime} \\
& N_{0}(x, y)=\gamma \\
& N_{0}(y, x)=\text { undefined. }
\end{aligned}
$$


If $\gamma$ is $2^{n}$-short then $N_{0}$ has $|\gamma|+1$ nodes $x_{0}, x_{1}, \ldots, x_{|\gamma|}$. Let $\gamma=\gamma_{0} \cdots \gamma_{l}$ (for some $l$, some $\gamma_{i} \in F$ for $i \leq l$ ) and let

$$
N_{0}\left(x_{i}, x_{j}\right)= \begin{cases}\frac{\gamma_{i} \gamma_{i+1} \cdots \gamma_{j-1}}{N_{0}\left(x_{j}, x_{i}\right)} & \text { if } i \leq j \\ \text { otherwise. }\end{cases}
$$

It is easy to check that $N_{0}$ is $2^{n}$-good.

By Lemma 5.21, in each round $\exists$ can play a $2^{r}$-good network if there are $r$ rounds left in the game. Since a $2^{r}$-good network is certainly consistent she will win the game if she plays this way.

\section{Ultraproducts and non-finite axiomatisability}

Theorem 6.1 (Łoś' theorem). Let $\left\{A_{i}: i \in I\right\}$ be a set of structures in a signature $L$ and let $D$ be an ultrafilter over $I$. For each $i \in I$ let $\overline{a_{i}}$ be an $n$-tuple of elements of $A_{i}$ and let $\bar{a}=\left[\left(\overline{a_{i}}: i \in I\right)\right]_{D}$. For any L-formula $\phi(\bar{x})$ where $\bar{x}$ is an n-tuple of variables,

$$
\Pi_{D} A_{i} \models \phi(\bar{a}) \Leftrightarrow\left\{i \in I: A_{i} \models \phi\left(\overline{a_{i}}\right)\right\} \in D .
$$

We are ready to prove our main theorem.

Proof. (Theorem 2.2) Consider $\mathcal{A}_{n}$ and let $\mathcal{A}_{n}^{\prime}$ be the SOM constructed from $\mathcal{A}_{n}$ in Definition 2.5. We saw in Lemma 2.8 that $\left(\mathcal{A}_{n}, \mathcal{A}_{n}^{\prime}\right) \models \Sigma$, for all $n \in \mathbb{N}$, where $\Sigma$ is the two-sorted theory given in Definition 2.7. Let $D$ be a non-principal ultrafilter over $\mathbb{N}$. Note that every cofinite subset of $\mathbb{N}$ belongs to $D$. By Loś' theorem $\Pi_{D}\left(\mathcal{A}_{i}, \mathcal{A}_{i}^{\prime}\right) \cong\left(\Pi_{D} \mathcal{A}_{i}, \Pi_{D} \mathcal{A}_{i}^{\prime}\right) \models \Sigma$. For $2^{r}<n \exists$ has a winning strategy in $G_{r}\left(\mathcal{A}_{n}\right)$ (Theorem 5.22) hence she also has a winning strategy in $G_{r}\left(\mathcal{A}_{n^{\prime}}\right)$ whenever $n^{\prime} \geq n$. By Proposition 3.3(4) there is a first-order formula $\sigma_{r}$ where $\mathcal{A}_{n^{\prime}} \models \sigma_{r}$ iff $\exists$ has a winning strategy in $G_{r}\left(\mathcal{A}_{n^{\prime}}\right)$. By Łoś' theorem, $\Pi_{D} \mathcal{A}_{i} \models \sigma_{r}$ for each $r \in \mathbb{N}$. By Proposition 3.3(4) again, $\exists$ has a winning strategy in $G_{r}\left(\Pi_{D} \mathcal{A}_{i}\right)$ for all $r \in \mathbb{N}$.

By Proposition 3.3(3) there is a countable $(X, Y)$ with $(X, Y) \equiv\left(\Pi_{D} \mathcal{A}_{i}, \Pi_{D} \mathcal{A}_{i}^{\prime}\right)$ such that $\exists$ has winning strategy in the infinite game $G(X)$. By Proposition 3.3(2), $X$ is an ordered monoid with a principal representation. By elementary equivalence $(X, Y) \models \Sigma$, and by Lemma $2.9, Y$ is a representable SOM.

Now, each $\mathcal{A}_{n}^{\prime}$ is an unrepresentable SOM (Theorem 4.11) but the non-principal ultraproduct $\Pi_{D} \mathcal{A}_{i}^{\prime}$ is elementarily equivalent to a representable SOM, $Y$. Suppose for contradiction that a finite number of axioms, wlog a single axiom $\sigma$, defines the class of representable SOMs. Then $\mathcal{A}_{n}^{\prime} \forall \models \sigma$ for all $n$, but $Y \models \sigma$. This is impossible, by Łoś' theorem.

The signature of ordered monoids is slightly less expressive than that of SOMs. In [9] it is proved that the class of all representable monoids is not finitely axiomatisable. 
Problem 6.2. Is the class of ordered monoids with principal representations elementary?

Note that Lemma 2.9 and Theorems 4.11 and 5.22 can be used to establish that this class is not finitely axiomatisable.

\section{Appendix}

This appendix contains a technical theorem and some lemmas from the paper (mainly about normal forms) and their proofs.

Lemma 6.3. Let $x, y \in(F \cup\{b\})^{*}$ and $x \geq y$. Then either $\operatorname{nf}(x) \stackrel{\psi \bar{\psi} \succ 1^{\prime}}{\longrightarrow} z \geq \operatorname{nf}(y)$ (for some $z$ and some $\psi \in\{f, \bar{g}\}$ ) or $\operatorname{nf}(y)$ is obtained from $\operatorname{nf}(x)$ by a sequence of reductions $(f g)^{n} \succ b$.

Proof. All the strings in the lemma belong to $(F \cup\{b\})^{*}$ so the only possible reductions are those in (I) and (II) from Definition 4.3, and the only replacements that we can apply from Definition 4.5 are (1)-(4).

Let $\operatorname{nf}(x)=x \geq y=\operatorname{nf}(y)$. There is a chain of reductions from $x$ to $y$

$$
x=x_{0} \stackrel{\tau_{0}}{\rightarrow} x_{1} \stackrel{\tau_{1}}{\rightarrow} \ldots \stackrel{\tau_{k-1}}{\rightarrow} x_{k}=y .
$$

Let us assume that there is no redundancy in this chain, in the following sense: there is no proper subsequence $\left(\sigma_{0}, \ldots, \sigma_{j-1}\right)$ of $\left(\tau_{0}, \tau_{1}, \ldots, \tau_{k-1}\right)$ such that there is a chain

$$
x=x_{0}^{\prime} \stackrel{\sigma_{0}}{\rightarrow} x_{1}^{\prime} \stackrel{\sigma_{1}}{\rightarrow} \cdots \stackrel{\sigma_{j-1}}{\rightarrow} x_{j}^{\prime}=y .
$$

Claim 1: Let $i \leq k$ and let $x_{i}$ be from (6). If there is no $j<i$ such that $\tau_{j}=\left(\psi \bar{\psi} \succ 1^{\prime}\right)$, where $\psi \in\{f, \bar{g}\}$, then $\operatorname{nf}\left(x_{i}\right)=x_{i}$.

To prove Claim 1, first note that $\operatorname{nf}\left(x_{0}\right)=x_{0}$. Now let $i>0$ and suppose inductively that $\operatorname{nf}\left(x_{i-1}\right)=x_{i-1}$. Now suppose, for contradiction, that $\operatorname{nf}\left(x_{i}\right) \neq x_{i}$. Since $x_{i} \in(F \cup\{b\})^{*}$ this implies that $x_{i}$ contains a substring $b \bar{g}, \bar{f} b, g \bar{g}$ or $\bar{f} f$ (see Definition 4.5).

Suppose $x_{i}=x^{0} b \bar{g} x^{1}$, for some $x^{0}, x^{1}$. Since $\operatorname{nf}\left(x_{i-1}\right)=x_{i-1}, x_{i-1}$ does not contain a substring $b \bar{g}$. So the reduction $\tau_{i-1}$ must have either replaced a string by something ending with $b$, replaced a string by something beginning with $\bar{g}$ or deleted a string between the $b$ and the $\bar{g}$. There are no reductions of the form $s \succ \bar{g} t$ (any $s, t$ ), so the second case is impossible. If a string between $b$ and $\bar{g}$ was deleted, then $\tau_{i-1}=\left(\psi \bar{\psi} \succ 1^{\prime}\right)$ for some $\psi$ and since $x_{i-1}$ does not contain $\bar{f} f$ or $g \bar{g}$ we must have $\psi \in\{f, \bar{g}\}$. But the assumption in the claim is that $\tau_{i-1}$ is not a reduction of this form. There is only one reduction to a string ending with $b$ and it is $(f g)^{n} \succ b$. But if $\tau_{i-1}=\left((f g)^{n} \succ b\right)$ then $x_{i-1}=x^{0}(f g)^{n} \bar{g} x^{1}$ so $\operatorname{nf}\left(x_{i-1}\right) \neq x_{i-1}$, contrary to assumption. 
If $x_{i}=x^{0} g \bar{g} x^{1}$ (for some $x^{0}, x^{1}$ ) then, as $x_{i-1}$ does not contain $g \bar{g}$, we must have one of the following: $\tau_{i-1}=(s \succ t g)$ or $\tau_{i-1}=(s \succ \bar{g} t)$ for some $s$, $t$, or $\tau_{i-1}=1^{\prime} \succ g \bar{g}$, or $\tau_{i-1}$ deleted a string $\psi \bar{\psi}$ where $\psi \in\{f, \bar{g}\}$. The last alternative (the deletion) can be ruled out using the assumption in the claim. Consulting Definition 4.3, we see that there are no reductions to a string starting with $\bar{g}$ or a string ending with $g$, so this case cannot happen. The 'reduction' $1^{\prime} \succ g \bar{g}$ is ruled out by assumption that there is no redundancy in the chain.

The cases where $x_{i}$ contains $\bar{f} b$ or $\bar{f} f$ are similar. Thus $x_{i}$ does not contain a substring $b \bar{g}, \bar{f} b, g \bar{g}$ or $\bar{f} f$ and so $\operatorname{nf}\left(x_{i}\right)=x_{i}$. This proves Claim 1 .

Suppose there is $i<k$ such that $\tau_{i}=\left(\psi \bar{\psi} \succ 1^{\prime}\right)$ with $\psi \in\{f, \bar{g}\}$ and let $i$ be the least such that $\tau_{i}$ is of this form. By Claim $1, x_{j}$ does not contain $b \bar{g}, g \bar{g}, \bar{f} b$ or $\bar{f} f$, for each $j<i$. We aim to show that $i$ can be taken to be 0 . Suppose $i>0$. We have $x_{i-1} \stackrel{\tau_{i-1}}{\longrightarrow} x_{i} \stackrel{\psi \bar{\psi} \succ 1^{\prime}}{\longrightarrow} x_{i+1}$.

Claim 2: If $x_{i-1} \stackrel{\tau_{i-1}}{\longrightarrow} x_{i} \stackrel{\psi \bar{\psi} \succ 1^{\prime}}{\longrightarrow} x_{i+1}$ then there is $\tau_{i}^{\prime}$ and $x_{i}^{\prime}$ such that $x_{i-1} \stackrel{\psi \bar{\psi} \succ 1^{\prime}}{\longrightarrow}$ $x_{i}^{\prime} \stackrel{\tau_{i}^{\prime}}{\rightarrow} x_{i+1}$.

To prove Claim 2 let $\tau_{i-1}=(s \succ t)$. If $t$ and $\psi \bar{\psi}$ are disjoint substrings of $x_{i}$ then clearly we can let $\tau_{i}^{\prime}=\tau_{i-1}$ and reverse the order of the two reductions. The only way that $t$ and $\psi \bar{\psi}$ are not disjoint in $x_{i}$ is if $t$ ends with $\psi$ or $t$ starts with $\bar{\psi}$. Let $\psi=f$ (the case $\psi=\bar{g}$ is similar). The only reductions $s \succ t$ where $t$ ends with $f$ or $t$ starts with $\bar{f}$ are $1^{\prime} \succ \bar{f} f$ and $b \bar{g} \succ(f g)^{n-1} f$. The (overlapping) sequence of reductions $x_{i-1} \stackrel{1^{\prime} \succ \bar{f} f}{\longrightarrow} x_{i} \stackrel{f \bar{f} \succ 1^{\prime}}{\longrightarrow} x_{i+1}$ contains a redundancy (since $x_{i-1}=x_{i+1}$ so we could delete both reductions) contrary to assumption. The case $\tau_{i-1}=\left(b \bar{g} \succ(f g)^{n-1} f\right)$ is contradicted by Claim 1. This proves the second claim.

Hence, if there is any $\tau_{i}$ in the chain of the form $\psi \bar{\psi} \succ 1^{\prime}$, for $\psi \in\{f, \bar{g}\}$ then by Claim 2 either $i=0$ or we can replace the chain (6) by another chain in which the reduction $\psi \bar{\psi} \succ 1^{\prime}$ occurs as the $(i-1)$ th reduction in the chain. Thus, this reduction can be moved to the front of the chain and so $x \stackrel{\psi \bar{\psi} \succ 1^{\prime}}{\longrightarrow} z \geq y$ (for some $z)$. This is the first alternative conclusion in the lemma.

Otherwise, none of the $\tau_{i}$ has the form $\psi \bar{\psi} \succ 1^{\prime}$, for $\psi \in\{f, \bar{g}\}$. By Claim 1, $\operatorname{nf}\left(x_{i}\right)=x_{i}$ for each $x_{i}$ in chain 6 . Hence, the only possibility for each reduction $\tau_{i}$ is $(f g)^{n} \succ b$ - any other reduction $x_{i} \stackrel{\tau_{i}}{\rightarrow} x_{i+1}$ would either be $\psi \bar{\psi} \succ 1^{\prime}(\psi \in\{f, \bar{g}\})$ or it would imply that $x_{i}$ contained $b \bar{g}, \bar{f} b, g \bar{g}$ or $\bar{f} f$ contradicting $\operatorname{nf}\left(x_{i}\right)=x_{i}$. This gives the second alternative conclusion in the lemma.

Lemma 6.4. Let $x, y, z \in(F \cup\{b\})^{*}$. Suppose $x=\operatorname{nf}(x)$ and $y z=\operatorname{nf}(y z)$ and that $y, z$ are minimal subject to $y ; z \geq x$ (i.e. if $y^{\prime} \leq y, z^{\prime} \leq z$ and $y^{\prime} z^{\prime} \geq x$ then $y^{\prime} \equiv y$ and $\left.z^{\prime} \equiv z\right)$. Then either 
- $y=y_{0} s, z=t z_{0}$, for some $y_{0}, z_{0}, s, t$ where $s t=(f g)^{i n}$ for some $i \in \mathbb{N}$, and $x=y_{0} b^{i} z_{0}$, or

- $y=y_{0} \phi, z=\bar{\phi} z_{0}$ and $y_{0} z_{0} \geq x$, for some $\phi \in F$ and some $y_{0}, z_{0}$.

Proof. Assume the conditions in the lemma. We have $\operatorname{nf}(y z) \geq \operatorname{nf}(x)$. By Lemma 5.1, either $y z \stackrel{\psi \bar{\psi} \succ 1^{\prime}}{\longrightarrow} \gamma \geq x$ (for some $\gamma$ and some $\psi \in\{f, \bar{g}\}$ ) or $\operatorname{nf}(x)$ is obtained from $\operatorname{nf}((y z))$ by a sequence of replacements $(f g)^{n} \succ b$. In the former case, the string $\psi \bar{\psi}$ in the reduction $\psi \bar{\psi} \succ 1^{\prime}$ cannot be confined to $y$ (or $z$ ) else $y$ (or $z$ ) is not minimal. So we must have $y=y_{0} \psi, z=\bar{\psi} z_{0}$ and $y_{0} z_{0} \geq x$, which is the second alternative conclusion in the lemma.

The other case is where $x$ is obtained from $y z$ by a sequence of replacements $(f g)^{n} \succ b$. We can group together adjacent substrings $(f g)^{n}$ of $y z$ that are reduced to $b$. So $x$ is obtained from $y z$ by replacing some substrings $(f g)^{i n}$ by $b^{i}$, where $i>0$. The substrings $(f g)^{i n}$ are not adjacent to each other.

If $(f g)^{i n}$ is replaced by $b^{i}$ then $(f g)^{i n}$ cannot be confined to $y$ (or $z$ ) else $y$ (or $z$ ) is not minimal. Hence $x$ is obtained from $y z$ by replacing a single substring $(f g)^{i n}$ by $b^{i}$ and this substring must be the concatenation of a non-empty final segment of $y$ and a non-empty initial segment of $z$. This gives the first alternative conclusion in the lemma.

Lemma 6.5. Let $e \geq 1^{\prime}$ and $\rho \in \mathcal{A}_{n}$.

(1) Either $k_{(f g)^{n}} e \geq k_{\Lambda} b$ or $e \equiv 1^{\prime}$ (and not both).

(2) Either $k_{\Lambda} b e \geq k_{(f g)^{n}}$ or $e \equiv 1^{\prime}$.

(3) If $\gamma \geq \rho k_{(f g)^{n}}$ but $\gamma \geq \rho k_{\Lambda} b$ then $\gamma \equiv \rho^{+} k_{(f g)^{n}}$ for some $\rho^{+} \geq \rho$.

(4) If $\gamma \geq \rho k_{\Lambda} b$ but $\gamma \geq \rho k_{(f g)^{n}}$ then $\gamma \equiv \rho^{+} k_{\Lambda} b$ for some $\rho^{+} \geq \rho$.

Proof. For (1), suppose $k_{(f g)^{n}} e \nsupseteq k_{\Lambda} b$. Note that $k_{(f g)^{n}} f \bar{f} \geq k_{\Lambda} b$ and $k_{(f g)^{n}} \bar{g} g \geq$ $k_{\Lambda} b$. Thus $e \geq 1^{\prime}$ must be obtained from the empty string by a series of insertions of $\bar{f} f \mathrm{~s}$ and $g \bar{g} \mathrm{~s}$. These insertions are all equivalences, so $e \equiv 1^{\prime}$. Part (2) is similar.

For (3), let $\gamma \geq \rho k_{(f g)^{n}}$. The only reductions in Definition 4.3 involving a character $k_{s}$ are listed as (III), (IV) and (V) and each has the form $k_{s} x \succ k_{t} y$ or $a x \succ k_{t}$, for some $s, t, x, y$. Hence if $x \geq \rho k_{s}$ then $x=x^{+} k_{t} y$ or $x=x^{+} a y$, for some $x^{+} \geq x$ and some $t, y$ such that $k_{t} y \geq k_{s}$. In the case under consideration we have $\gamma=\rho^{\prime} \kappa$ for some $\rho^{\prime} \geq \rho$ and some $\kappa \geq k_{(f g)^{n}}$.

Since $\kappa \geq k_{(f g)^{n}}$ there is a chain $\kappa \stackrel{\tau_{0}}{\rightarrow} \cdots \stackrel{\tau_{i}}{\rightarrow} \rho k_{(f g)^{n}}$. The only possible reductions $s \stackrel{\tau_{i}}{\rightarrow} e k_{(f g)^{n}} e^{\prime}$ (any $\left.e, e^{\prime} \geq 1^{\prime}\right)$ are (a) $\tau_{i}=\left(a b \succ k_{(f g)^{n}}\right)$, (b) $\tau_{i}=\left(k_{(f g)^{n-1} f} g \succ\right.$ $\left.k_{(f g)^{n}}\right)$ or $(\mathrm{c}) \tau_{i}=\left(\phi \bar{\phi} \succ 1^{\prime}\right)$. Now $a b \geq k_{\Lambda} b$ so we can rule out reduction (a). Also, $k_{(f g)^{n-1} f} g \equiv k_{\Lambda}(f g)^{n} \geq k_{\Lambda} b$ (use Lemma 4.4), so we we can rule out reduction (b). Thus all the reductions in the chain are of the type (c), which means that all the strings in the chain have the form $e k_{(f g)^{n}} e^{\prime}$, so $\kappa=e k_{(f g)^{n}} e^{\prime}$ for some $e, e^{\prime} \geq 1^{\prime}$. 
By part (1), since $\kappa \geq k_{\Lambda} b$, we have $e^{\prime} \equiv 1^{\prime}$. Thus $\kappa \equiv e k_{(f g)^{n}}$, for some $e \geq 1^{\prime}$ and $\gamma=\rho^{\prime} e k_{(f g)^{n}}$. Let $\rho^{+}=\rho^{\prime} e$ to get the required result.

Part (4) is similar.

Theorem 6.6. Let $x, y \in \mathcal{A}_{n}$ and $\phi \in F$.

(1) $x \leq y \Rightarrow x / \phi \leq y / \phi-i$.e. for each $z \in y / \phi$ there is $z^{\prime} \in x / \phi$ with $z^{\prime} \leq z$.

(2) $y ; \phi \geq x \Leftrightarrow y \geq x / \phi-i$.e. there is $z \in x / \phi$ with $z \leq y$.

(3) $(x ; y) / \phi \leq x ;(y / \phi)$ - i.e. for all $z \in y / \phi$ there is $z^{\prime} \in(x ; y) / \phi$ with $z^{\prime} \leq x ; z$.

Proof. (1): First let $\phi \in\{\bar{f}, g\}$, so $x / \phi$ and $y / \phi$ are defined by case I of Definition 5.5: $x / \phi=\operatorname{nf}(x \bar{\phi})$ and $y / \phi=\operatorname{nf}(y \bar{\phi})$. So if $x \leq y$ then $x / \phi \equiv x \bar{\phi} \leq y \bar{\phi} \equiv y / \phi$.

Now let $\phi=f$, so $x / \phi$ and $y / \phi$ are defined by case II of Definition 5.5. Suppose $y \geq_{k} x$. We deal with the case $k=1$, i.e. $x$ is obtained from $y$ by a single reduction. The general case will then follow by a simple induction over $k$. So assume $y \geq_{1} x$. If $y \equiv x$ then, by Lemma $4.8, y=x$ and so $y / f=x / f$. So we assume that $y<x$. This eliminates several possibilities for the single reduction $y \geq_{1} x$. Now we break into the two possibilities in the definition of $y / f$ (case II). If $y \neq y_{0} f$ (for any $y_{0}$ ) then $y / f=y \bar{f} \geq x \bar{f} \geq z^{\prime}$, for all $z^{\prime} \in x / f$ by Lemma 5.6. If $y=y_{0} f$ for some $y_{0}$, we see from Definition 4.5 that there are three possibilities. First, $x=x_{0} f$ and $y_{0} \geq x_{0}$. Then $x / f=\left(x_{0} f\right) / f=x_{0}$ and $y / f=\left(y_{0} f\right) / f=y_{0}$ and we are done. Second, if $x=x_{0} \phi^{\prime}$, for some $\phi^{\prime} \leq f$, then as $f$ is minimal with respect to $<$ we have $\phi^{\prime}=f$, so the reduction is in fact an equivalence, contrary to assumption. Finally, any other possible reduction $\tau$ with $y_{0} f \stackrel{\tau}{\rightarrow} x$ must involve a non-empty final segment of $y_{0}$ concatenated with a non-empty initial segment of $f$. But any such reduction must be an equivalence. This completes the proof for case II.

Similarly we can prove case III, where $\phi=\bar{g}$ and $y \geq_{1} x$. If $y=y_{0} k_{\Lambda}(f g)^{n-1} f$ (for some $y_{0}$ ) then $y / \bar{g}=\left\{y_{0} k_{\Lambda} b, y_{0} k_{(f g)^{n}}\right\}$. Since $k_{\Lambda}(f g)^{n-1} f$ is minimal with respect to $<$, we have $x=x_{0} k_{\Lambda}(f g)^{n-1} f$ for some $x_{0} \leq y_{0}$ and $x / \bar{g}=$ $\left\{x_{0} k_{\Lambda} b, x_{0} k_{(f g)^{n}}\right\}$ and the lemma holds true. Next suppose $y=y_{0} \bar{g}$ for some $y_{0}$ and $y / \bar{g}=y_{0}$. From Definition 4.5 we must have $x=x_{0} \bar{g}$ and $y_{0} \geq x_{0}$. Then Lemma 5.7 gives us the result. If $y=y_{0} k_{s} f$, for some $y_{0}$ and some initial segment $s f$ of $(f g)^{n}$ then, as in the proof of case II, we have $x / \bar{g} \leq y / \bar{g}$, as required. Finally, suppose $y \neq y_{0} k_{\Lambda}(f g)^{n-1} f, y_{0} \bar{g}, y_{0} k_{s} f$, for any $y_{0}$ and any initial segment $s f$ of $(f g)^{n}$. Then $\left.y / \bar{g}=(y ; g)\right\rfloor$. Either $\left.(y ; g)\right\rfloor=y ; g$ or $y=y_{0}(f g)^{n-1} f$, for some $y_{0}$, and $\left.(y ; g)\right\rfloor=y_{0} b$. By Lemma 5.6 there is $z \in x / \bar{g}$ with $z \leq x g$. If $(y ; g)\rfloor=y ; g$ then there is $z \in x / \bar{g}$ with $z \leq x ; g \leq(y ; g)\rfloor=y / \bar{g}$, as required. Else $y=y_{0}(f g)^{n-1} f$ (for some $y_{0}$ ) and, since $(f g)^{n-1} f$ is minimal with respect to $<$, we have $x=x_{0}(f g)^{n-1} f$ for some $x_{0} \leq y_{0}$. Hence $x / \bar{g}=x_{0} b \leq y_{0} b=y / \bar{g}$.

(2): The right to left implication holds, by Lemma 5.6. For the left to right implication we must show that if $y ; \phi \geq x$ then there is $z \in x / \phi$ with $y \geq z$. By 
Lemma 5.7, there is $z^{\prime} \in(y ; \phi) / \phi$ with $z^{\prime} \leq y$. Since $x \leq y ; \phi$, the first part of the theorem says that there is $z \in x / \phi$ with $z^{\prime} \geq z$. So there is $z \in x / \phi$ with $z \leq z^{\prime} \leq y$.

(3): This easily follows by the second part of the theorem.

\section{REFERENCES}

[1] H. Andréka, On the 'union-relation composition' reducts of relation algebras, Abstracts Amer. Math. Soc. 10 (1989), 174. Full, unpublished manuscript called 'On the representation problem of distributive semilattice-ordered semigroups', Mathematical Institute, Hungarian Academy of Sciences, Budapest.

[2] H. Andréka, Representation of distributive lattice-ordered semigroups with binary relations, Algebra Universalis 28 (1989), 12-25.

[3] H. Andréka, S. Givant and I. Németi, Decision problems for equational theories of relation algebras, Bulletin of Section of Logic 23 (1994), 47-52.

[4] H. Andréka and I. Németi, Axiomatization of identity-free equations valid in relation algebras, Algebra Universalis 35 (1996), 256-264.

[5] D. Bredikhin, Abstract characteristic of some classes of algebras of relations, Algebra and theory of numbers 2 (1977), 3-19. (Russian)

[6] D. Bredikhin, The equational theory of relation algebras with positive operations, Izv. Vyash. Uchebn. Zaved. Math. 3 (1993), 23-30. (Russian)

[7] D. Bredikhin and B. Schein, Representation of ordered semigroups and lattices by binary relations, Colloquium Mathematicum 39 (1978), 1-12.

[8] M. Haiman, Arguesian lattices which are not type 1, Algebra Universalis 28 (1991), 128-137.

[9] R. Hirsch, The class of representable ordered monoids has a recursively enumerable, universal theory but it is not finitely axiomatisable, Logic Journal of the IGPL 13 (2005), $159-172$.

[10] R. Hirsch and I. Hodkinson, Representability is not decidable for finite relation algebras, Trans. Amer. Math. Soc. 353 (2001), 1403-1425.

[11] R. Hirsch and I. Hodkinson, Relation algebras by games, North-Holland. Elsevier Science, Amsterdam, 2002.

[12] I. Hodkinson and Sz. Mikulás, Axiomatizability of reduct of algebras of relations, Algebra Universalis 43 (2000), 127-156.

[13] I. Hodkinson and Y. Venema, Canonical varieties with no canonical axiomatisation, Trans. Amer. Math. Soc. 357 (2005), 4579-4605.

[14] B. Jónsson, The theory of binary relations, in: Algebraic logic, H Andréka, J Monk, and I Németi, eds., vol. 54 of Colloq. Math. Soc. J. Bolyai, North-Holland, Amsterdam, 1991 pp. 245-292.

[15] R. McKenzie, The representation of relation algebras, $\mathrm{PhD}$ thesis, University of Colorado at Boulder, 1966.

[16] Sz. Mikulás, Axiomatizability of algebras of binary relations, in: Classical and New Paradigms of Computation and their Complexity Hierarchies, B Löwe, B Piwinger, and T Räsch, eds., Kluwer Academic Publishers, 2004, pp. 187-205.

[17] J. Monk, On representable relation algebras, Michigan Mathematics Journal 11 (1964), $207-210$.

[18] A. Tarski, On the calculus of relations, J. Symbolic Logic 6 (1941), 73-89. 
[19] A. Tarski, Contributions to the theory of models, III, Koninkl. Nederl. Akad. Wetensch Proc. 58 (= Indag. Math. 17) (1955), 56-64.

[20] A. Tarski and S. Givant, A formalization of set theory without variables, Number $\mathbf{4 1}$ in Colloquium Publications. Amer. Math. Soc., Providence, Rhode Island, 1987.

ROBIN HIRSCH

Computer Science, UCL, Gower Street, London WC1E 6BT, UK

e-mail:r.hirsch@cs.ucl.ac.uk

Szabolcs Mikulás

Computer Science and Information Systems, Birkbeck College, Malet Street, London WC1E $7 \mathrm{HX}$, UK

e-mail: szabolcs@dcs.bbk.ac.uk 